\title{
Dual-Specificity Phosphatase 1 (DUSP1) Has a Central Role in Redox Homeostasis and Inflammation in the Mouse Cochlea
}

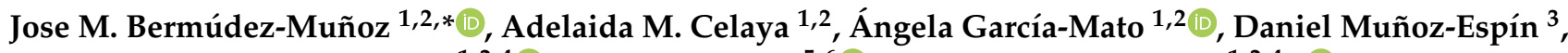

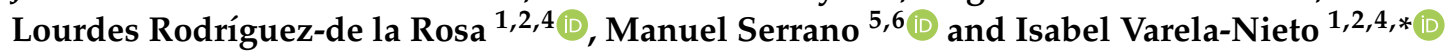

Citation: Bermúdez-Muñoz, J.M.; Celaya, A.M.; García-Mato, Á.; Muñoz-Espín, D.; Rodríguez-de la Rosa, L.; Serrano, M.; Varela-Nieto, I. Dual-Specificity Phosphatase 1 (DUSP1) Has a Central Role in Redox Homeostasis and Inflammation in the Mouse Cochlea. Antioxidants 2021, 10 1351. https://doi.org/10.3390/ antiox10091351

Academic Editors: Anna Rita Fetoni and Jing Wang

Received: 28 June 2021

Accepted: 23 August 2021

Published: 25 August 2021

Publisher's Note: MDPI stays neutral with regard to jurisdictional claims in published maps and institutional affiliations.

Copyright: (c) 2021 by the authors. Licensee MDPI, Basel, Switzerland. This article is an open access article distributed under the terms and conditions of the Creative Commons Attribution (CC BY) license (https:/ / creativecommons.org/licenses/by/ $4.0 /)$.
1 Institute for Biomedical Research "Alberto Sols", Spanish National Research Council-Autonomous University of Madrid (CSIC-UAM), 28029 Madrid, Spain; acelaya@iib.uam.es (A.M.C.); agarciamato@iib.uam.es (Á.G.-M.); lrodriguez@iib.uam.es (L.R.-d.1.R.)

2 Rare Diseases Networking Biomedical Research Centre (CIBERER), CIBER, Carlos III Institute of Health, 28029 Madrid, Spain

3 CRUK Cambridge Centre Early Detection Programme, Department of Oncology, University of Cambridge, Hutchison/MRC Research Centre, Cambridge CB2 0XZ, UK; dm742@cam.ac.uk

4 Hospital La Paz Institute for Health Research (IdiPAZ), 28029 Madrid, Spain

5 Institute for Research in Biomedicine, Barcelona Institute of Science and Technology (BIST), 08028 Barcelona, Spain; manuel.serrano@irbbarcelona.org

6 Catalan Institution for Research and Advanced Studies (ICREA), 08010 Barcelona, Spain

* Correspondence: jmbermudez@iib.uam.es (J.M.B.-M.); i.varela.nieto@csic.es (I.V.-N.); Tel.: +34-91-585-4422 (I.V.-N.)

\begin{abstract}
Stress-activated protein kinases (SAPK) are associated with sensorineural hearing loss (SNHL) of multiple etiologies. Their activity is tightly regulated by dual-specificity phosphatase 1 (DUSP1), whose loss of function leads to sustained SAPK activation. Dusp1 gene knockout in mice accelerates SNHL progression and triggers inflammation, redox imbalance and hair cell (HC) death. To better understand the link between inflammation and redox imbalance, we analyzed the cochlear transcriptome in Dusp1 $1^{-/-}$mice. RNA sequencing analysis (GSE176114) indicated that Dusp1 $1^{-/-}$ cochleae can be defined by a distinct profile of key cellular expression programs, including genes of the inflammatory response and glutathione (GSH) metabolism. To dissociate the two components, we treated $D u s p 1^{-/-}$mice with $\mathrm{N}$-acetylcysteine, and hearing was followed-up longitudinally by auditory brainstem response recordings. A combination of immunofluorescence, Western blotting, enzymatic activity, GSH levels measurements and RT-qPCR techniques were used. N-acetylcysteine treatment delayed the onset of SNHL and mitigated cochlear damage, with fewer TUNEL ${ }^{+} \mathrm{HC}_{\text {and }}$ lower numbers of spiral ganglion neurons with $\mathrm{p}-\mathrm{H} 2 \mathrm{AX}$ foci. $\mathrm{N}$-acetylcysteine not only improved the redox balance in Dusp $1^{-/-}$mice but also inhibited cytokine production and reduced macrophage recruitment. Our data point to a critical role for DUSP1 in controlling the cross-talk between oxidative stress and inflammation.
\end{abstract}

Keywords: hearing; N-acetylcysteine; glutathione; antioxidants; reactive oxygen species (ROS); mitochondria; apoptosis; inflammation; RNAseq

\section{Introduction}

Progressive sensorineural hearing loss (SNHL) is a bilateral and gradual impairment of hearing as a consequence of cochlear degeneration, most commonly resulting from the death of mechanosensitive hair cells (HCs) or/and spiral ganglion neurons (SGNs), which are both irreplaceable in mammals. Genetic inheritance in combination with environmental and lifestyle factors determines the onset, severity and progression of SNHL [1], which is the most frequent sensory impairment in the elderly. The World Health Organization estimates that over 700 million people worldwide will experience disabling hearing loss by 2050 [2]. Preventive therapies to suppress the molecular mechanisms responsible for HC and SGN death are the most promising treatment options to avert irreversible damage. 
Oxidative stress and mitochondrial dysfunction are common triggering factors for cochlear degeneration in all scenarios causing SNHL [3]. Accordingly, a large number of compounds have been tested for their ability to reduce reactive oxygen species (ROS) generation, promote the ROS scavenger system, or enhance cochlear antioxidant defenses Among them, N-acetylcysteine (NAC) is one of the most widely investigated, and it is administered in mice by either intraperitoneal injection or oral gavage $(12.5-400 \mathrm{mg} / \mathrm{kg})[4,5]$. NAC oral intake is approved for human use in the range $200-1200 \mathrm{mg} /$ day [4]. NAC can stimulate, indirectly, glutathione (GSH) synthesis, acting as L-cysteine precursor, and can also directly break disulfide bonds, acting as a reducing agent [6]. While the use of antioxidants to restore the redox imbalance in the cochlea has proved to be effective to some extent, in vitro and in vivo experiments revealed that the elevated levels of ROS represent just one component of a more complex and orchestrated phenomenon involving impaired mitochondrial quality control and function, loss of proteostasis, DNA damage and genomic instability, apoptosis, inflammation and altered intercellular communication $[7,8]$. Progress in this area is, accordingly, dependent on identifying as many molecular targets as possible in the aberrant regulatory pathways. In this context, dual-specificity phosphatase 1 (DUSP1) emerges as a central candidate.

DUSP1 is an inducible MAP kinase phosphatase (MKP), a branch of the dual specificity phosphatase family that regulates mitogen-activated protein kinase (MAPK) activation [9]. MAPKs are specific Ser/Thr kinases that operate in a three-tier kinase cascade to integrate signals from extracellular stimuli to direct physiological responses, including proliferation, differentiation, motility, inflammation and/or survival. MAPKs integrate multiple highly heterogeneous extracellular signals, including growth factors, mitogens or cytokines secreted from neighboring cells, as well as environmental stressors such as oxidative stress, UV radiation, heat or osmotic shock. JNK1/2/3 and p38 isoforms (MAPK11/12/13/14) are the major MAPKs that participate in transducing stress stimuli and, hence, are known as stress-activated protein kinases (SAPKs) [10]. SAPKs have been reported as mediators of the cochlear stress response to aminoglycoside ototoxicity, noise insult and aging both in vitro and in vivo $[7,11,12]$. Because the overactivation of SAPKs leads to cell death, their inhibition has been reported to promote cell survival and hearing protection after cochlear insult in humans [13] and mice [14]. The spatiotemporal activation of SAPKs leads to cellular fate-shifting outcomes, and thus, it is tightly regulated by feedback loops that are ultimately based on the dephosphorylating capacity of DUSP1. DUSP1 is induced by stress stimuli to control the magnitude and extent of SAPK activation $[9,15]$. It is also able, albeit with less affinity, to dephosphorylate ERK1/2 [15]. Accordingly, DUSP1 can be considered as a converging node for several signal transduction pathways, participating in multiple physiological processes [16] that include cytokine production, macrophage recruitment and inflammation resolution [17]. Mouse DUSP1 has also been reported to have a role in glucocorticoid-mediated inflammatory repression [18], limiting cytokine production $[19,20]$ and inhibiting NF- $K \beta$ [21].

We recently showed that mice with genetic deficiency of Dusp 1 present with exacerbated inflammation and generalized HC and SGN loss along aging, thus providing a good model of premature SNHL [9]. Indeed, DUSP1 is key in the control of the progression of SNHL and in the response to noise damage mediated by increased activity of p38 $\alpha$ [9]. Here, we aim to deepen the understanding of the role of DUSP1 in hearing loss, which we began to unveil in our previous paper [9]. The comparative RNAseq analysis of the cochleae of wild type and mutant Dusp 1 knockout mice shown in this manuscript sustained our hypothesis that oxidation secondary to DUSP1 loss of action had a role in triggering premature hearing loss. We consider that if the redox imbalance was significant, treatment with an antioxidant should prevent hearing loss. NAC was used because its antioxidant properties are well-known and its human use approved [4]. Therefore, NAC was used as a pharmacological tool to prevent redox imbalance. An in-depth knowledge of the molecular bases of SNHL with regard to DUSP1 involvement should contribute to the development of effective therapies. In this regard, the main results of the present study are 
the following: (i) comparative RNA sequencing (RNAseq) data analysis of wild-type (WT) and Dusp $1^{-1-}(\mathrm{KO})$ mouse cochleae revealed the aberrant expression of genes involved in GSH metabolism, inflammation, DNA damage, apoptosis and neuronal homeostasis in Dusp $1^{-/-}$mice; (ii) antioxidant supplementation (NAC) in Dusp $1^{-/-}$mice partially recovers hearing thresholds, likely by restoring the cellular antioxidant system and limiting cochlear damage; (iii) cochleae of NAC-treated Dusp $1^{-/-}$mice show preserved mitochondrial integrity and limited ROS production, thus preventing a chronic inflammatory state; and (iv) DNA damage and apoptosis are reduced in SGNs and HCs, respectively, in NAC-treated Dusp $1^{-/-}$mice.

\section{Materials and Methods}

\subsection{Mice}

$D u s p 1^{+/+}(\mathrm{WT}), D u s p 1^{-/-}(\mathrm{KO})$ and NAC-treated Dusp $1^{-/-}(\mathrm{KO}+\mathrm{NAC})$ mice were used in the study ( $n=25$ for WT and KO, $n=15$ for $\mathrm{KO}+\mathrm{NAC}$ ). Mice were generated on a mixed 129S2/SvPas:C57BL/6 genetic background and were genotyped as described [22]. No sex-linked differences were noted between genotypes in hearing; thus, male and female mice were analyzed together.

\subsection{Antioxidant Treatment}

For drug administration, we used the protocol of a previous study [23], with some modifications. Briefly, fresh NAC was prepared every week at a dose of $10 \mathrm{~g} / \mathrm{L}$ in drinking water $(\mathrm{pH} \sim 7.4)$ and was administered for 13 weeks, beginning treatment at weaning (week 4) up to 4 months of age (week 16). Animals were maintained under controlled temperature and light-dark cycle conditions and received standard chow diet and water ad libitum. To assess the impact of antioxidant treatment on auditory function, hearing thresholds were evaluated by the auditory brainstem response (ABR) at the end of weeks 8 and 16. WT and KO mice were sacrificed, and samples were collected at both time points, whilst $\mathrm{KO}+\mathrm{NAC}$ only at the second time point.

\subsection{Hearing Evaluation}

Animals were anesthetized and placed cater-cornered in a sound-attenuating chamber for recording in open field configuration $10 \mathrm{~cm}$ apart from the calibrated MF1 speaker. ABR testing was performed using a RZ6-A-P1 processor (Tucker-Davis Technologies, Alachua, FL, USA). Electrical auditory processing was collected in response to TDT click (broadband) and tone burst $(4,8,16,24$ and $32 \mathrm{kHz})$ stimuli. All stimuli were presented at $31 \mathrm{pps}$ rate with $10 \mathrm{~ms}$ acquisition time and averaged 1000 or 750 times for the click and pure tone stimuli, respectively. The response was recorded essentially as reported [24], in 5 to $10 \mathrm{~dB}$ steps from maximum ( $90 \mathrm{~dB}$ SPL) to minimum (15-20 dB SPL) amplitude, and analyzed using BioSigRZ software 5.6.0 (Tucker-Davis Technologies, Alachua, FL, USA). The lowest intensity (SPL level) that evoked a recognizable ABR five-peak wave pattern was established as the hearing threshold for each stimulus. ABR waves I, II, III and IV and inter-peak I-II, II-IV and I-IV latencies were analyzed at fixed 70-dB SPL click stimulation. Wave I amplitude and latency were further analyzed at all intensity levels.

\subsection{RNA-Sequencing}

Inner ear dissection was performed as described [25] and samples were frozen in RNAlater ${ }^{\circledR}$ solution (Ambion, Foster City, CA, USA). Cochlear RNA was extracted using the RNeasy Plus Mini kit (Qiagen, Hilden, Germany) automated on the Qiacube (Qiagen, Hilden, Germany). For RNA sequencing, $1 \mu \mathrm{g}$ of total RNA from cochlear RNA extracts from 20- and 32-week-old mice was used ( $n=3$ per group). The average sample RNA integrity number was 9.2-9.7 (2100 Bioanalyzer, Agilent Technologies, Palo Alto, CA, USA). The polyA+ fraction was purified and randomly fragmented, converted to double-stranded cDNA and processed through subsequent enzymatic treatments of end-repair, dA-tailing, and ligation to adapters using the Illumina TruSeq Stranded mRNA Sample Preparation 
Kit (Part \#15031047 Rev. D; San Diego, CA, USA). This kit incorporates dUTP during the 2nd strand cDNA synthesis, meaning that only the cDNA strand generated during 1st strand synthesis is sequenced. The adapter-ligated library was completed by PCR using Illumina PE primers (8 cycles). The resulting purified cDNA library was applied to an Illumina flow cell for cluster generation and was sequenced on the Illumina HiSeq2000 platform. Single-end (50-bp) sequenced reads were analyzed with the next presso pipeline (http:/ / ubio.bioinfo.cnio.es/people/ograna/nextpresso/, accessed on 1 May 2017). Sequencing quality was checked with FastQC 0.10.1 (http:/ / www.bioinformatics.babraham. ac.uk/projects / fastqc/, accessed on 1 May 2017). Reads were aligned to the mouse genome (NCBI37/mm9) with TopHat-2.0.10 [26] using Bowtie 1.0.0 [27] and Samtools 0.1.19 [28], allowing 2 mismatches and 5 multi-hits. Transcript quantification and differential expression were calculated with Cufflinks 2.2.1 [26], using the mouse NCBI37/mm9 transcript annotations from https: / / ccb.jhu.edu/software/tophat/igenomes.shtml, accessed on 28 June 2021. Gene-set enrichment analysis (GSEA) [29] was conducted on Reactome, BioCarta and KEGG pathways; the RNAseq gene list was pre-ranked by statistic scores, setting 'gene set' as the permutation method and with 1000 permutations. Only those gene sets with significant enrichment levels (false discovery rate [FDR] $q$-value $<0.05$ ) were considered. GSEA results were visualized using Enrichment Map [30] and AutoAnnotate [31] applications for Cytoscape 3.8.2 [32], following a described pipeline [33] and applying an FDR cutoff of 0.01 for a total of 158 enriched processes or pathways (Figure 1 and Table S1). Heatmaps were plotted using the Heatmapper web-enabled tool [34]. RNAseq data discussed in this publication have been deposited on NCBI's Gene Expression Omnibus and are accessible through GEO Series accession number GSE176114.

\subsection{RT-qPCR}

Inner ear dissection and RNA extraction was performed as described above. Quality determination and cDNA generation from pooled cochlear RNA extracts (3 cochlea from different animals per group) were performed as reported [35]. Quantitative amplification was performed in triplicate on a Quant Studio 7 Flex PCR System (Applied Biosystems, Foster City, CA, USA) using either commercial TaqMan probes or gene specific primers (Table S2 and [25]). Data were collected after each amplification step and analyzed with QuantStudio $^{\mathrm{TM}}$ Real-Time PCR software 1.3 (Applied Biosystems). Hprt1 gene was used as a housekeeping gene, and the n-fold differences were calculated using the $2^{-\Delta \Delta \mathrm{Ct}}$ method.

\subsection{Cochlear Morphology and Immunohistochemistry}

Sample processing for histological analysis has been previously described [25]. For cochlear cytoarchitecture, inner ear paraffin cochlear cross sections $(5 \mu \mathrm{m})$ from 3 mice per experimental group were stained with hematoxylin-eosin, analyzed with a Zeiss Axiophot microscope (Carl Zeiss, Jena, Germany) and photographed with an Olympus DP70 digital camera (Melville, NY, USA). For immunohistofluorescence, $10 \mu \mathrm{m}$ cryostat cross sections $(n=3)$ were incubated with cytochrome c oxidase subunit I (1:100, \#PA5-26688; Molecular Probes, Eugene OR, USA), p-H2A.X (1:100, \#2577; Cell Signaling Technology, Danvers, MA, USA) or IBA1 (1:100, \#Ab5076; Abcam, Cambridge, UK). A confocal laser-scanning (Zeiss LSM710, Carl Zeiss, Jena, Germany) microscope was used to acquire stack images. For each cochlear turn, total IBA1 intensity and mean cytochrome c oxidase subunit I intensity in the spiral ligament and spiral ganglion, respectively, were computed with Fiji software [36] from at least 3 mice of each genotype in 4 serial sections. p-H2A.X foci were counted in 5-10 neuronal nuclei per section in slides containing 4 serial sections from at least 3 mice of each genotype using the Fiji 3D objects Counter plugin in confocal stack images. To differentiate positive p-H2A.X foci from larger and less intense auto-fluorescent cytoplasmatic stress granules in neurons, high pass intensity (value $=160)$ and voxel size $(\min =3, \max =70)$ thresholds were applied. 


\subsection{Hair Cell Number Quantification and TUNEL Assay}

Inner ear samples were processed, dissected and stained with Myo7a (1:150, \#PT-256790; Proteus, Ramona, CA, USA), and the TdT-mediated dUTP nick-end labeling (TUNEL) (Dead-End Fluorometric TUNEL System, Promega, Madison, WI, USA) assay was used for HC quantification and apoptosis valuation, as described [25]. Low magnification images of organ of Corti (OC) half turns were taken with a Nikon 90i epifluorescence microscope (Nikon Corp., Tokyo, Japan) and cochleograms were plotted using a custom Fiji plugin for at least $3 \mathrm{OC}$ whole mounts per experimental group. $\mathrm{HC}$ number and $\mathrm{TUNEL}^{+}$outer hair cells (OHCs) were counted in $200 \mu \mathrm{m}$ of the basilar membrane in the apical, basal and middle regions located $15-25 \%, 50-60 \%$ and $70-80 \%$, respectively, from the apex. TUNELpositive $\mathrm{OHCs}$ were scored as a percentage of the total number of $\mathrm{OHC}$. Representative stack images were acquired with a Zeiss LSM710 confocal laser-scanning microscope (Carl Zeiss, Jena, Germany) at the specified cochlear regions.

\subsection{Protein Extraction and Immunoblotting}

Whole cochlear pooled protein extracts were prepared from 3 mice, as described [35]. A fixed volume of extract was separated by SDS-PAGE and transferred to PVDF membranes $(0.2 \mu \mathrm{m}$, Bio-Rad Laboratories, Hercules, CA, USA) using the Bio-Rad Trans Blot TURBO apparatus. Prior to antibody incubation (overnight $4{ }^{\circ} \mathrm{C}$ ), membranes were blocked with $5 \%$ bovine serum albumin or non-fat dried milk in $0.1 \%$ Tween, $1 \mathrm{mM}$ TBS. Primary antibodies used were as follows: rabbit anti-P-p38 (1:1000, \#9211), anti-P-JNK (1:1000, \#4668), anti-PAKT (1:1000, \#9271) and anti-P-H2AX (1:1000, \#2577) (all from Cell Signaling Technology, Danvers, MA, USA), rabbit anti-P22phox (1:250, \#sc-20781; Santa Cruz Biotechnology, Dallas, TX, USA), anti-HO-1 (1:1000, \#AB1284; Merck, Darmstadt, Germany), anti-MnSOD (1:1000, \#06-984; Millipore, Merck, Darmstadt, Germany), goat anti-NQO1 (1:1000, \#ab2346; Abcam) and rabbit anti-PI3K (1:15000, in-house). Immunocomplexes were visualized with peroxidase-conjugated secondary antibodies ( $1 \mathrm{~h}$ at room temperature), and bands were detected using the Clarity ${ }^{\mathrm{TM}}$ Western ECL Substrate (Bio-Rad) on an Image Quant LAS4000 mini digital camera (GE Healthcare Bio-Sciences, Pittsburgh, PA, USA). Band densities were quantified in triplicate using Image Quant TL software 8.1 (GE Healthcare Bio-Sciences, Pittsburgh, PA, USA ).

\subsection{Protein Carbonylation}

Carbonylation of cochlear proteins was measured using the Oxyblot ${ }^{\mathrm{TM}}$ Kit (Millipore, Merck, Darmstadt, Germany). In brief, one aliquot of pooled protein extracts from each experimental group was derivatized with 2,4-dinitrophenylhydrazine (DR, derivatization reaction) and a second aliquot was treated with control solution (NC, negative control). Oxidized proteins were detected using first a primary antibody specific to the dinitrophenylhydrazone residues and then an HRP-conjugated secondary antibody. Protein extraction, electrophoresis and immunodetection were performed in triplicate as outlined above.

2.10. Measurement of Glucose-6-Phosphate Dehydrogenase, 6-Phosphogluconate Dehydrogenase and Glutathione Reductase Activity

Mitochondrial and cytosolic fractions from two inner ears (cochlea and vestibuli) per experimental group were isolated as described [25]. Glucose-6-phosphate dehydrogenase (G6PD) and 6-phosphogluconate dehydrogenase (PGD) activity were measured in cytosolic fractions [37], and glutathione reductase (GSR) activity was measured in both cytosolic and mitochondrial fractions using the Glutathione Reductase Assay Kit (Sigma-Aldrich, Madrid, Spain). All spectrophotometric measurements were performed at least in triplicate in a 96-well format in a VERSA max Tunable Microplate Reader (Molecular Devices, Sunnyvale, CA, USA). Protein concentrations were determined using the DC Protein Assay kit (Bio-Rad Laboratories, Hercules, CA, USA ). 


\subsection{Glutathione and Glutathione Disulfide Analysis}

Measurement of GSH and glutathione disulfide (GSSG) levels was performed as described [38]. Briefly, two inner ears per experimental group were homogenized in extraction buffer ( $0.1 \%$ Triton X-100, $0.6 \%$ sulfosalicylic acid, $5 \mathrm{mM}$ EDTA, $0.1 \mathrm{M}$ potassium phosphate $\mathrm{pH}$ 7.5) on ice using a Dounce tissue grinder (Wheaton, Millville, NJ, USA) and centrifuged at $8000 \times g$ at $4{ }^{\circ} \mathrm{C}$ for $10 \mathrm{~min}$. Then, $20 \mu \mathrm{L}$ of supernatant was used for total GSH measurement or was incubated for $1 \mathrm{~h}$ at room temperature with 2-vinylpyridine for GSSG measurement, prior to addition of DTNB (Ellman's reagent), glutathione reductase and $\beta$-NADPH solutions. The reaction was measured spectrophotometrically at $412 \mathrm{~nm}$ every $30 \mathrm{~s}$ for $2 \mathrm{~min}$, and samples were run in triplicate in a 96-well format in a VERSA max Tunable Microplate Reader (Molecular Devices). TNB (5'-thio-2-nitrobenzoic acid) formation rates were calculated to determine total GSH and GSSG levels using linear regression from the standard curve plots. GSH concentration was obtained by subtracting GSSG from total GSH concentration. Protein concentrations were determined using the DC Protein Assay kit (Bio-Rad Laboratories, Hercules, CA, USA).

\subsection{Statistical Analysis}

Sample size was estimated to obtain a $90 \%$ statistical power with a significance level of 0.05 , using data from previous experiments and calculating Cohen's d value. Data analysis was performed with IBM SPSS 25.0 (IBM Corp., Armonk, NY, USA). Statistical significance was assessed by one-way analysis of variance (ANOVA) after the Shapiro-Wilk and Levene test for determination of normal distribution of data and equality of variances, respectively. Bonferroni or Tamhane tests were used as appropriate when differences were obtained. Results were considered statistically significant at a $p$-value $<0.05$.

\section{Results}

\subsection{Dusp1 Deficiency Affects Cellular Programs Involved in Cochlear Homeostasis}

We carried out RNAseq analysis of Dusp $1^{+/+}$and Dusp $1^{-/-}$cochleae in 20- and 32week-old mice. RNAseq datasets were used to run GSEA (Figure 1 and Table S1). Of the 158 enriched pathways in 20-week-old Dusp $1^{-/-}$cochlea, 41 pathways were directly related to the immune system, and 17 were also enriched in 32-week-old mice. Similarly, 21 DNArelated pathways were enriched in 20-week-old Dusp1 $1^{-/}$cochleae, and 12 (including DNA repair pathways) were also enriched in the 32-week-old mouse cochlear samples. Intrinsic and extrinsic apoptotic pathways were also enhanced at 20 but not at 32 weeks. Moreover, 18 pathways related to neural homeostasis were negatively enriched in $\mathrm{Dusp} 1^{-/-}$cochleae at 20 weeks, as compared with Dusp $1^{+/+}$cochleae, and eight of them (including neuronal system and glutamatergic synapse pathways) remained negatively enriched at 32 weeks. We also observed gene enrichment for the oxidative phosphorylation process in 20-weekold Dusp $1^{-/-}$cochleae, which pointed to an increase in mitochondrial ROS production by a highly active electron transport chain. By contrast, the mitochondrial biogenesis program driven by the peroxisome proliferator-activated receptor $\gamma$ coactivator $1 \alpha$ (PGC1A) pathway was negatively enriched, suggesting the reduced generation of new mitochondrial content and impaired organelle dynamics and function. Of note, these two phenomena correlated with the enhancement of the antioxidant GSH metabolism pathway in 20week-old Dusp1 $1^{-/-}$mice, which was maintained at 32 weeks of age (Figure 2A), possibly to compensate/overcome mitochondrial dysfunction and oxidative damage. Overall, these results indicate that deficiency in DUSP1 activity in mice impacts cochlear redox homeostasis, mitochondrial quality control, apoptosis and inflammation. 


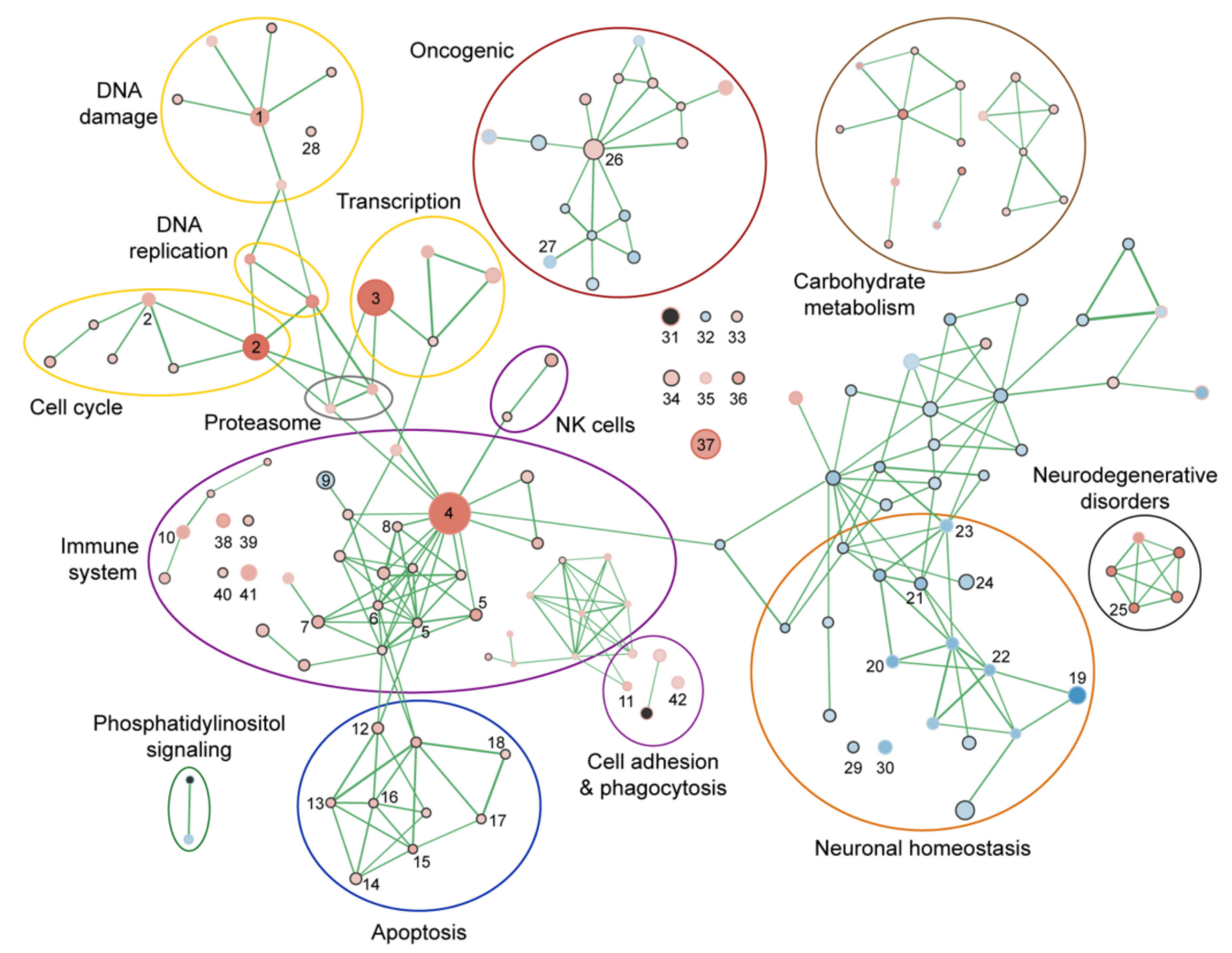

1. DNA repair (Reactome)
2. Cell cycle (Reactome \& KEGG)
3. Gene expression (Reactome)
4. Immune system (Reactome)
5. NF-k $\beta$ signaling (KEGG \& BioCarta)
6. Stress pathway (BioCarta)
7. TNF signaling (KEGG)
8. IL1R pathway (BioCarta)
9. MAPK signaling (KEGG)
10. Cytokine-cytokine receptor interaction (KEGG)
11. Phagosome (KEGG)
12. Apoptosis (KEGG)
13. Death pathway (BioCarta)
14. Programmed cell death pathway (Reactome)
15. Caspase pathway (BioCarta)
16. Mitochondria pathway (BioCarta)
17. FAS pathway (BioCarta)
18. TNFR1 pathway (BioCarta)
19. Neuronal system (Reactome)
20. Glutamatergic synapse (KEGG)
21. Cholinergic synapse (KEGG)

22. Gabaergic synapse (KEGG)
23. Dopaminergic synapse (KEGG)
24. Calcium signaling (KEGG)
25. Oxidative phosphorylation (KEGG)
26. Pathways in cancer (KEGG)
27. Hippo signaling (KEGG)
28. ATM pathway (BioCarta)
29. Synaptic vesicle cycle (KEGG)
30. Axon guidance (KEGG)
31. Organelle biogenesis and maintenance (Reactome)
32. PGC1A pathway (BioCarta)
33. PPARA pathway (BioCarta)
34. AMPK signaling (KEGG)
35. Glutathione metabolism (KEGG)
36. Peroxisome (KEGG)
37. Metabolism of proteins (Reactome)
38. Leucocyte transendothelial migration (KEGG)
39. IL2RB pathway (BioCarta)
40. IL12 pathway (BioCarta)
41. Chemokine signaling (KEGG)
42. FCYR mediated phagoytosis (KEGG)

Gene set size (number of genes)
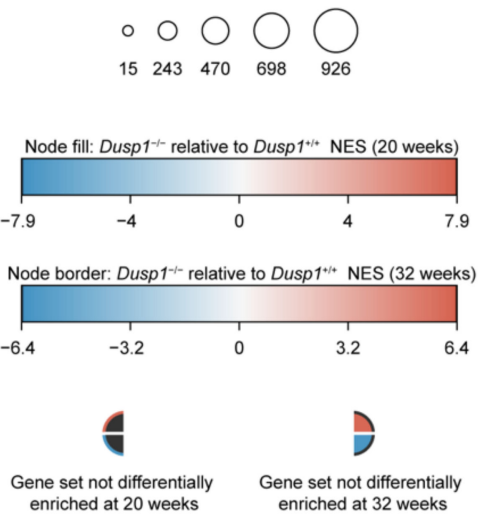

Figure 1. Gene-set enrichment analysis of RNAseq data sets from 20- and 32-week-old Dusp $1^{+/+}$and Dusp $1^{-/-}$cochlea. Enrichment analysis visualization for identification of altered gene sets (nodes). Gene sets derive from Reactome, KEGG and BioCarta databases. Node size denotes gene set number. Differentially enriched gene sets in 20 -week-old Dusp $1^{-/-}$mice are designated by node fill while node border designates 32-week-old data. Normalized enrichment value (NES) for each time point is illustrated in a color scale from blue (negative enrichment) to red (positive enrichment). No representation of the gene set at either time point is shown by a grey node fill or grey border, respectively. Connection between nodes is represented by lines. Line width symbolizes number of genes shared by both nodes. Most interesting nodes are numbered and listed (1-42). Related nodes are grouped in modules and labeled accordingly. Related modules share the same color. 
A

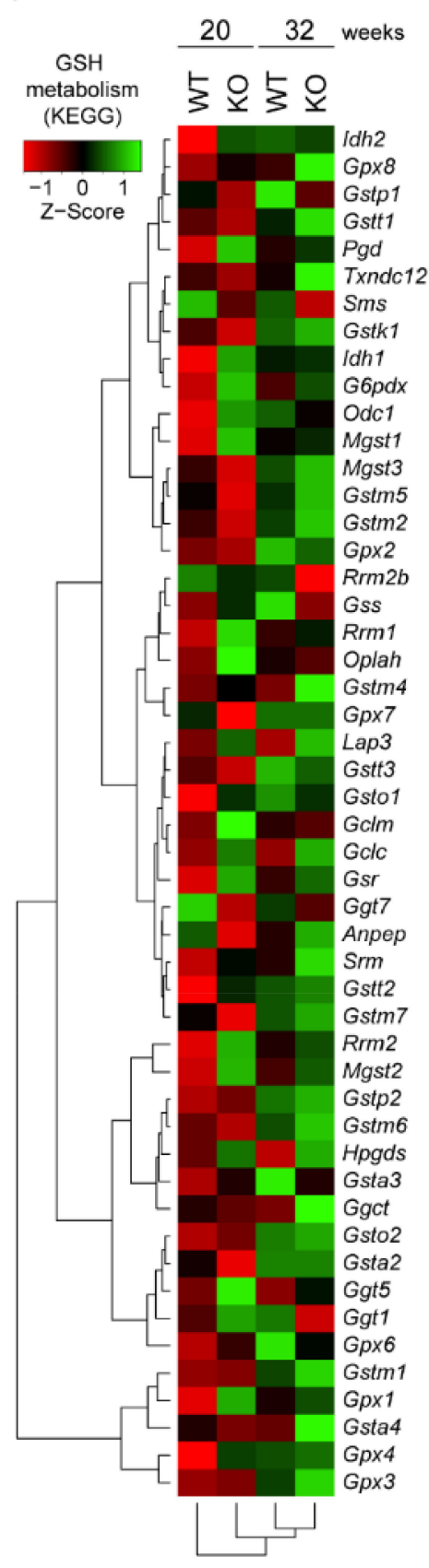

B

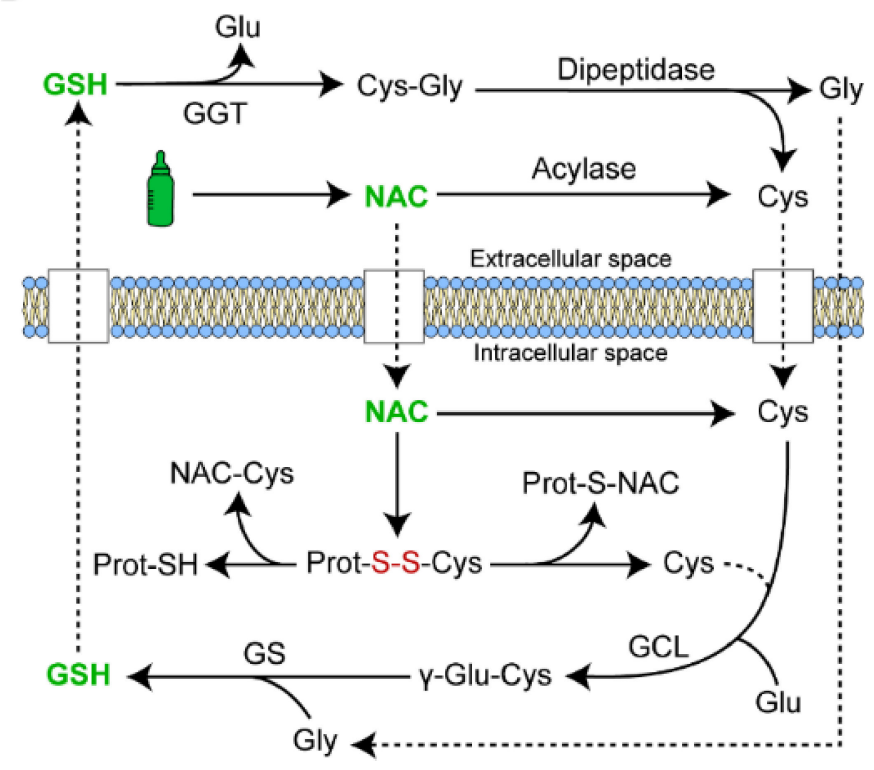

C

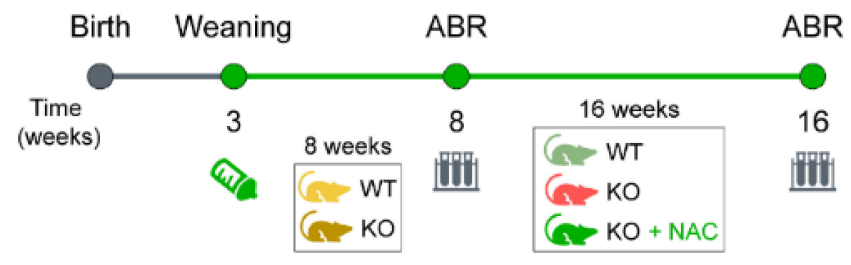

D
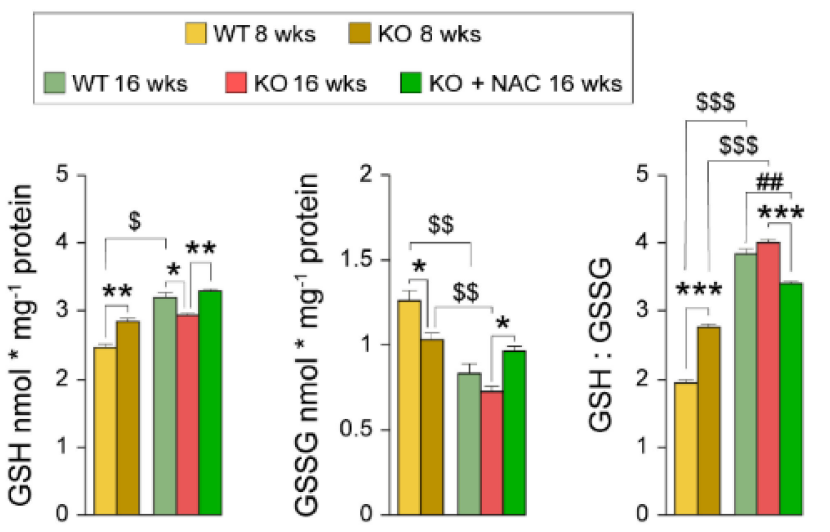

E

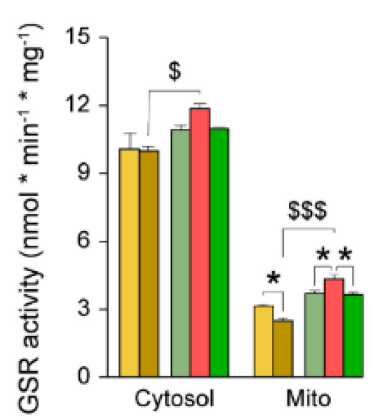

$\mathrm{F}$

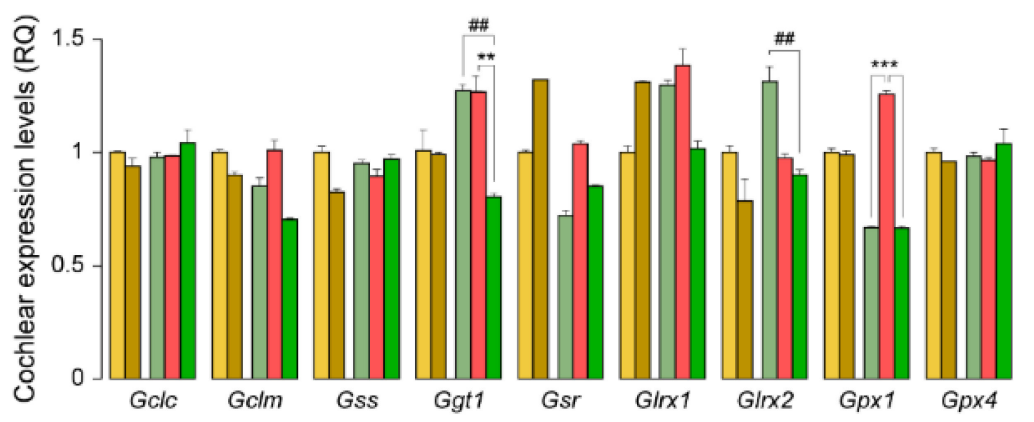

Figure 2. Impact of $\mathrm{N}$-acetylcysteine administration for glutathione metabolism. (A) Glutathione (GSH) metabolism (KEGG database) heatmap generated from differential expression data derived from RNAseq. (B) Scheme illustrating GSH biosynthesis, extracellular recycling and N-acetylcysteine (NAC) antioxidant power. GSH and NAC appear in green; glutathione 
synthesis and recycling enzymes appear in black capital letters: GCL (glutamate-cysteine ligase), GS (glutathione synthetase), GGT (gamma glutamyl transferase); glutathione precursors: Cys (cysteine), Glu (glutamate) and Gly (glycine); oxidized protein disulfide bonds in red (S-S). (C) Experimental design illustrating the start and end-time points of NAC administration (weaning-16 weeks), hearing evaluation (auditory brainstem response [ABR]) testing time points (8 and 16 weeks), experimental groups and sampling time points. (D) GSH, glutathione disulfide (GSSG) levels and GSH:GSSG ratio from pooled samples of 2 inner ears of 8- and 16-week-old wild-type (WT), Dusp1 knock-out (KO) and NAC-treated Dusp1 KO mice. All measurements were performed in triplicate. GSH and GSSG values are presented relative to milligram of protein. (E) GSR (glutathione reductase) activity in cytosolic and mitochondrial fractions from pooled samples of 2 inner ears per condition. Measurements were performed in triplicate, and values are presented relative to milligram of protein. (F) RT-qPCR gene expression levels of GSH-related enzymes from whole cochlea pooled samples from 3 mice per condition. Expression levels were calculated as $2^{-\Delta \Delta C t}(\mathrm{RQ})$ from triplicate measurements using Hprt1 as a reference gene and normalized to levels in 8-week-old WT mice. All data are presented as mean $\pm \mathrm{SEM}$. Statistical significance between genotypes and time points was analyzed by one-way ANOVA: * vs. KO, \# vs. WT, \$ 8-week-old mice vs. 16-week-old mice $\left({ }^{*}, \$ p<0.05 ;{ }^{* *}, \# \#, \$ \$ p<0.01 ;{ }^{* *}, \$ \$ \$ p<0.001\right)$.

\subsection{N-Acetylcysteine Treatment of Dusp1 $1^{-/-}$Mice Has a Positive Impact on Cochlear Glutathione Metabolism}

We hypothesized that the gene upregulation of the GSH metabolism pathway in Dusp $1^{-/-}$cochleae might be a consequence of increased ROS production by overfunctioning mitochondria that likely drives early and progressive hearing loss in Dusp $1^{-/-}$ mice. Accordingly, enhancing GSH synthesis with NAC might be a strategy to counteract redox imbalance (Figure $2 \mathrm{~B})$. To test this, we supplied NAC (10 g/L in drinking water) to 3-week-old Dusp1 ${ }^{-1-}$ mice for 13 weeks and then evaluated hearing loss progression by $\mathrm{ABR}$ in the three experimental groups-WT, KO and $\mathrm{KO}+\mathrm{NAC}$ - at 8 and 16 weeks of age. Samples were collected at both time points for untreated groups and only at the end time point for NAC-treated Dusp1 $1^{-/-}$mice (Figure 2C). We found that GSH levels were higher and GSSG levels were lower in 8-week-old Dusp1-/- inner ears than in equivalent Dusp $1^{+/+}$inner ears, resulting in a higher GSH:GSSG ratio (Figure 2D). These differences were not sustained in 16-week-old Dusp1-/- mice, which showed lower levels of reduced and oxidized forms than Dusp $1^{+/+}$mice and similar GSH:GSSG ratio levels (Figure 2D). At this time point, inner ear GSH and GSSG levels were higher in NAC-treated Dusp1-/mice than in untreated $D u s p 1^{-/-}$mice but were not significantly different from those in Dusp $1^{+/+}$mice. Inner ear GSR activity in both cytosolic and mitochondrial extracts was lower in 16-week-old WT and $\mathrm{KO}+\mathrm{NAC}$ groups than in equivalent $\mathrm{KO}$ groups (Figure 2E). GSR activity in both compartments increased from 8 to 16 weeks of age in the untreated KO group (Figure 2E).

Finally, RT-qPCR analysis of genes related to cochlear GSH synthesis revealed that expression of the antioxidant enzyme glutathione peroxidase 1 (Gpx1) was higher in the untreated $\mathrm{KO}$ group than in the WT and $\mathrm{KO}+\mathrm{NAC}$ groups (Figure 2F). An eventual higher GPX1 activity would require, from the higher GSR activity observed in Dusp1-/mice, maintaining the GSH pool and catalyzing the hydrogen peroxide reduction. Of note, NAC treatment specifically reduced the cochlear expression of the GSH recycling enzyme, gamma glutamyl transferase 1 (Ggt1), as compared with untreated groups (Figure 2F).

\subsection{N-Acetylcysteine Treatment Delays Hearing Loss Onset and Reduces Hair Cell Death in Dusp1-/- Mice}

We next performed hearing tests in the three experimental groups at 8 and 16 weeks of age. ABR analysis showed that NAC administration protected against hearing loss in Dusp1 $1^{-/-}$mice, with intermediate hearing thresholds between those of untreated Dusp $1^{-/-}$mice and Dusp $1^{+/+}$mice (Figure 3A). Specifically, NAC-treated Dusp $1^{-/-}$mice and $D u s p 1^{+/+}$mice displayed lower hearing thresholds for the click and tone burst stimuli than Dusp $1^{-/-}$mice at 8 and 16 weeks of age, although with evident age-related differences illustrated by the characteristic progressive high frequency hearing loss pattern in all mouse groups. NAC-mediated protection at the end-time point was evident for the frequencies 
located in the middle regions of the basilar membrane $(8,16$ and $24 \mathrm{kHz})$ and when the cochleae were multifrequency stimulated (click stimulus). Threshold shifts between both time points (Figure S1A) suggested that the antioxidant power provided by NAC was particularly effective in the first weeks of treatment, as evidenced by the faster sound conduction in 8-week-old NAC-treated Dusp1 ${ }^{-/-}$, but not later, as revealed by ABR latency analysis (Figure S1B-D).

A
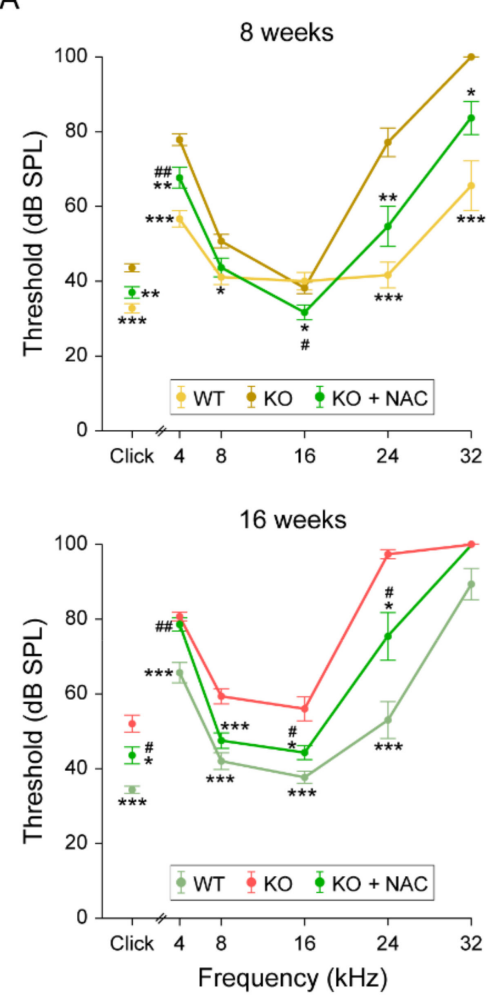

B

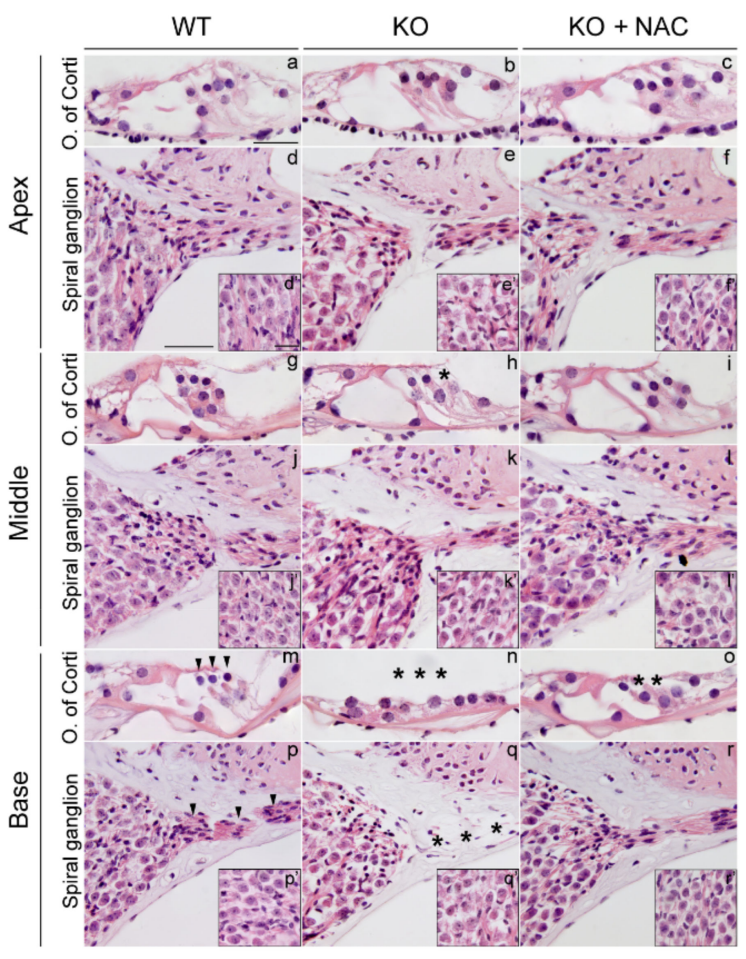

C

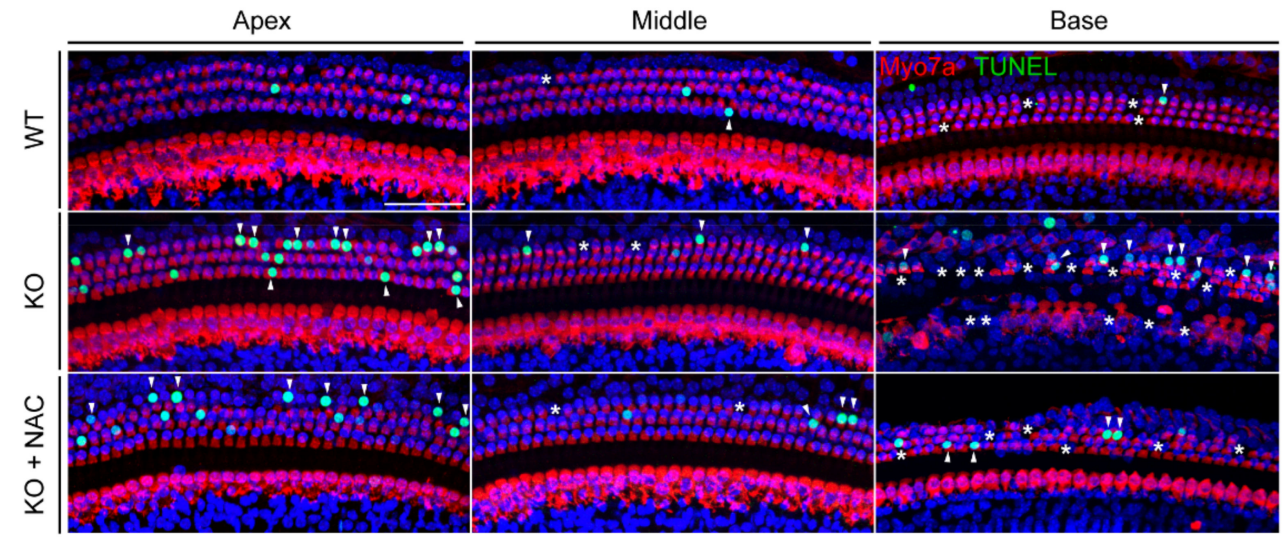

D

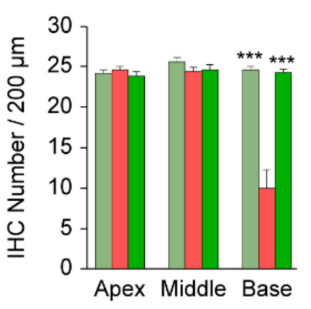

E

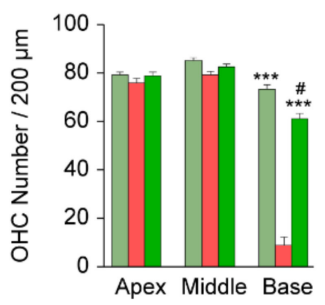

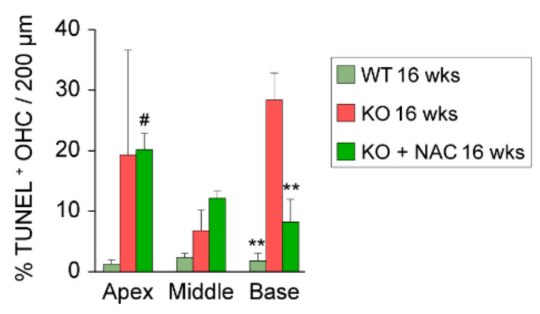

Figure 3. Comparative hearing evaluation, cochlear cytoarchitecture and organ of Corti degeneration. (A) Audiograms representing click and tone burst stimuli $(4,8,16,24$ and $32 \mathrm{kHz})$ auditory brainstem response (ABR) thresholds of 8- and 
16-week-old wild-type (WT), Dusp1 knock-out (KO) and NAC-treated Dusp1 KO mice. Data are presented as mean \pm SEM of at least 15 mice per condition. Statistical significance between genotypes was analyzed by one-way ANOVA: * vs. KO, \# vs. WT $\left({ }^{*}, \# p<0.05 ;{ }^{* *}\right.$, \#\# $\left.p<0.01 ;{ }^{* * *} p<0.001\right)$. (B) Representative basal, middle and apical turns hematoxylin-eosin stained cochlear mid-modiolar microphotographs of the organ of Corti (OC) (a-c, g-i and m-o) and spiral ganglion (SG) $(d-f, j-1$ and $p-r)$. SG close-ups $\left(d^{\prime}-f^{\prime}, j^{\prime}-l^{\prime}\right.$ and $\left.p^{\prime}-r^{\prime}\right)$. Scale bars: $25 \mu \mathrm{m}$ in a, $50 \mu \mathrm{m}$ in $\mathrm{d}$ and $25 \mu \mathrm{m}$ in $\mathrm{d}^{\prime}$. Asterisks and arrowheads indicate the absence or presence, respectively, of hair cells and fibers. (C) Representative confocal images of TUNEL-stained (green) basal, middle and apical basilar membrane regions of 16-week-old WT, Dusp1 KO and NAC-treated Dusp1 KO mice co-immunolabeled with Myo7a (red). Asterisks and arrowheads indicate hair cell (HC) loss and TUNEL ${ }^{+}$ nuclei, respectively. Scale bar: $50 \mu \mathrm{m}$. (D) Inner (IHC) and outer (OHC) hair cell number per $200 \mu \mathrm{m}$ of basal, middle or apical basilar membrane regions. (E) Percentage of TUNEL ${ }^{+}$OHCs in $200 \mu \mathrm{m}$ of basal, middle or apical basilar membrane sections. Data are presented as mean \pm SEM of 3 mice per condition. Statistical significance between genotypes was analyzed by one-way ANOVA: ${ }^{*}$ vs. KO, \# vs. WT $\left({ }^{*}, \# p<0.05 ;{ }^{* *}, \# \#<0.01 ;{ }^{* * *} p<0.001\right)$.

In accordance with the hearing evaluation findings, histological analysis of cochlear sections revealed better preservation of the OC and SGNs in the basal and middle turns of 16-week-old NAC-treated Dusp $1^{-/-}$mice than of untreated Dusp $1^{-/}$mice (Figure 3B). No anatomical alterations were observed in cochleae of $D u s p 1^{+/+}$mice, whereas untreated $D u s p 1^{-/-}$mice displayed a flat OC and neuronal fiber degeneration in the basal turn. NAC-treated Dusp $1^{-/-}$mice exhibited basal turn OHC loss with no alterations in the SG. Untreated-Dusp $1^{-/-}$mice showed patchy OHC loss in the middle turn that was not observed in the other two groups, as expected from the ABR analysis. Next, we performed TUNEL assays and HC Myo7a immunostaining in OC whole mounts (Figure 3C). HC counts confirmed the alterations observed by hematoxylin-eosin staining, with significant inner (IHC) and outer (OHC) loss in the basal turn cochlea of Dusp1 $1^{-/}$mice as compared with NAC-treated Dusp $1^{-/-}$and $D u s p 1^{+/+}$mice (Figure 3D). Accordingly, the percentage of apoptotic OHCs in the basal turn was higher in untreated Dusp1-/- mice than in the other two groups (Figure 3E).

\subsection{N-Acetylcysteine Administration Modulates Redox Systems and Limits Reactive Oxygen Species Production in Dusp $1^{-/-}$Cochleae}

To better understand how NAC treatment modulates the cochlear antioxidant defense and ROS production systems in Dusp1 $1^{-/-}$mice, we studied the expression levels of key redox genes. Gene expression analysis showed that Nrf2, Nqo1, Sod2 and Prdx6 levels were higher in 16-week-old Dusp $1^{+/+}$mice than in younger mice (Figure $4 \mathrm{~A}$ ); however, the overall expression profile indicated that NAC administration did not stimulate antioxidant gene expression, which was similar between untreated and NAC-treated Dusp1 $1^{-/-}$mice. In fact, the expression of several genes (Keap1, Sirt1, Sesn2 and G6pd) was lower in cochleae of NACtreated Dusp $1^{-/-}$mice than in equivalent untreated mice when compared with $D u s p 1^{+/+}$ mice, whereas Ho1 expression was lower in NAC-treated cochleae than in the other two groups. By contrast, P22phox, a component of the $\mathrm{O}_{2}{ }^{--}$generating NADPH oxidase (NOX) complex, increased in 16-week-old Dusp $1^{-/-}$mice, and this was not evident in equivalent NAC-treated Dusp1 $1^{-/-}$mice, which had levels similar to those of Dusp $1^{+/+}$mice. The same expression pattern was observed for Ucp2, an important mitochondrial carrier that controls mitochondria-derived ROS generation in the respiratory chain and whose expression is stimulated upon oxidative stress. The cochlear expression of Ucp1 was preserved by NAC treatment in Dusp1 $1^{-/-}$mice as compared with untreated $D u s p 1^{-/-}$and $D u s p 1^{+/+}$mice, as both latter groups showed a marked reduction in expression when compared with levels in 8-week-old mice. UCP1 generates heat by leaking protons to the mitochondrial matrix and increasing mitochondrial permeability. Finally, the cochlear expression of the nitric oxide isoforms $i$ Nos and $n$ Nos was higher in 16-week-old Dusp $1^{+/+}$mice than in 8-week-old counterparts and was unaffected (iNos) or lower ( $n N o s)$ in equivalent Dusp1 $1^{-/}$with or without NAC supplementation (Figure 4B). 
A

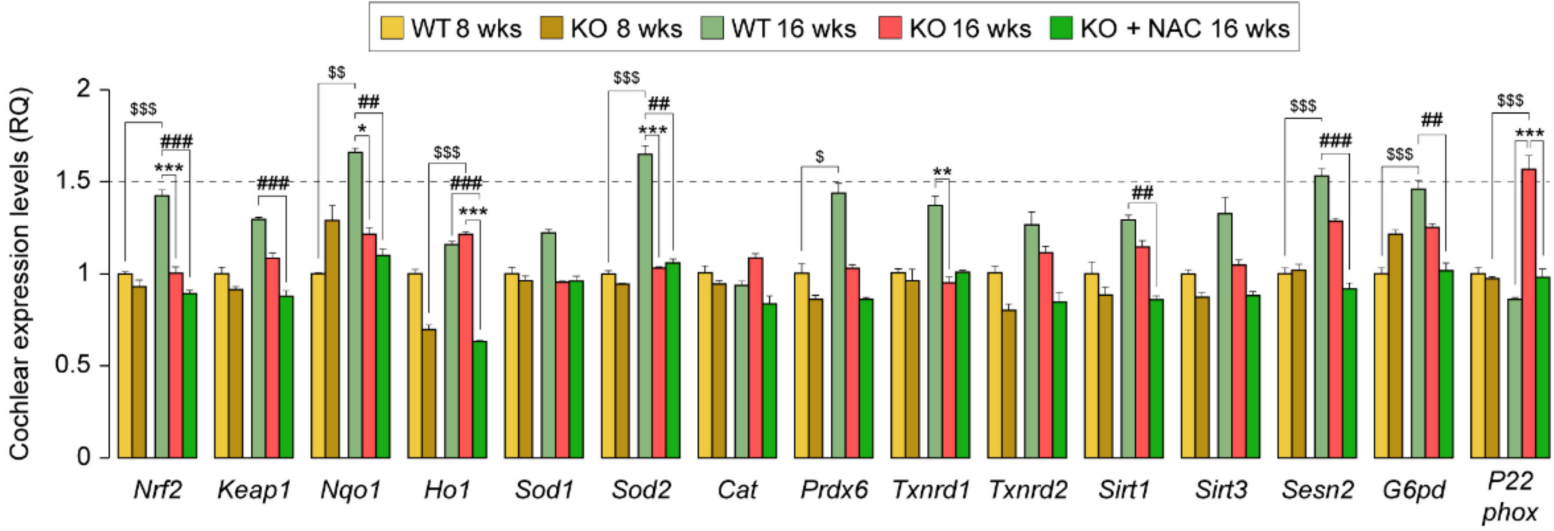

B

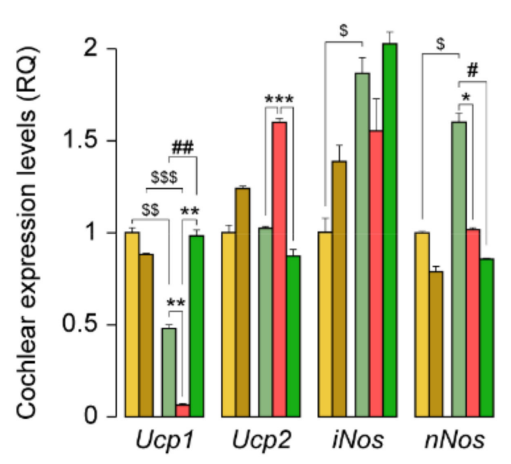

C

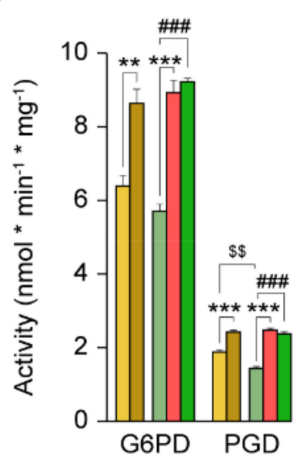

D

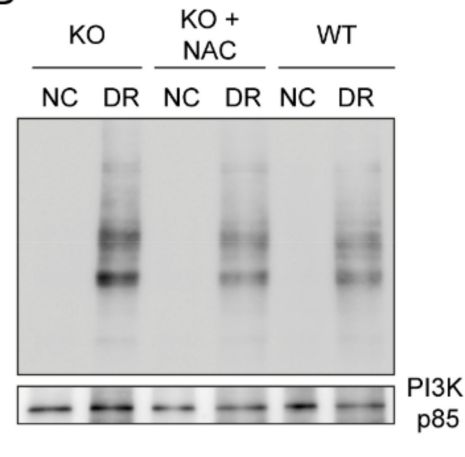

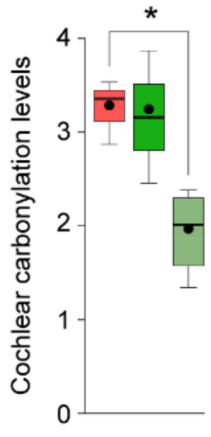

$\mathrm{E}$

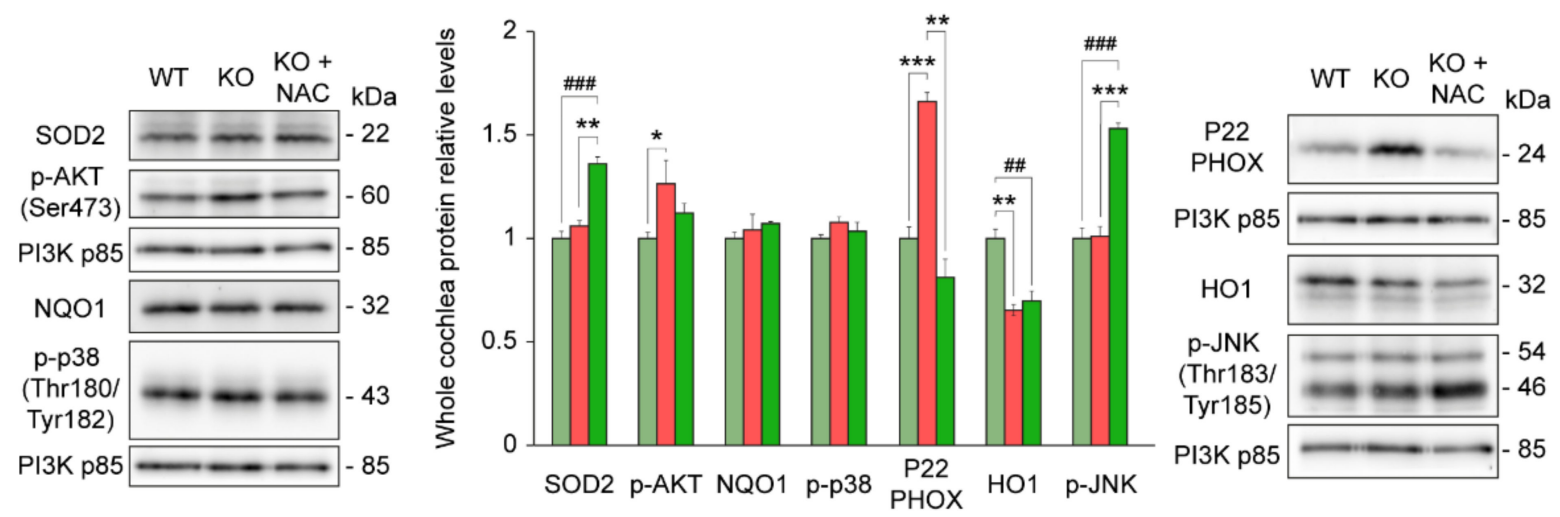

Figure 4. Impact of N-acetylcysteine administration for cochlear redox homeostasis. (A,B) RT-qPCR gene expression levels of redox enzymes from whole cochlea pooled samples from 3 mice per condition. Expression levels were calculated as $2^{-\Delta \Delta \mathrm{Ct}}(\mathrm{RQ})$ from triplicate measurements using $H$ prt1 as a reference gene and normalized to levels in 8-week-old wild-type (WT) mice. Data are presented as mean \pm SEM. (C) Glucose-6-phosphate dehydrogenase (G6PD) and 6-phosphogluconate dehydrogenase (PGD) activity from pooled inner ear cytosolic fractions of two mice per condition. Measurements were performed in triplicate, and values are presented relative to milligram of protein. Data are presented as mean \pm SEM. (D) Cochlear oxidative protein carbonylation levels of pooled cochlear protein extracts from 3 mice per condition analyzed with the Oxyblot ${ }^{\mathrm{TM}}$ Kit. Derivatized protein extracts (DR) are shown together with non-derivatized extracts (NC) in the representative blot image. Quantification was performed in triplicate, and levels were referred to those of PI3K. Data are presented as a box plot, mean value is plotted as a filled circle and whiskers represent min and max values. (E) Western blotting of pooled cochlear protein extracts from 3 mice per condition. All protein levels are referred to those of PI3K and normalized to data from 16-week-old WT mice. Data are presented as mean \pm SEM. Statistical significance between genotypes and time points was analyzed by one-way ANOVA: * vs. KO, \# vs. WT, \$ 8-week-old mice vs. 16-week-old mice $\left({ }^{*}, \#, \$ p<0.05 ; * *\right.$, \#\#,\$ $\left.p<0.01 ; * * * \# \#, \$ \$ p<0.001\right)$. 
Antioxidant defense, particularly the GSH system, is highly dependent on the reductive power of NADPH, which is produced mainly in the cytosol by sequential reactions catalyzed by G6PD and PGD. Enzymatic measurements revealed higher activity of both enzymes in 8- and 16-week-old untreated and NAC-treated Dusp $1^{-/-}$mice than in Dusp $1^{+/+}$ mice (Figure $4 \mathrm{C}$ ). We also assessed the extent of oxidative damage by analyzing protein carbonylation, finding that NAC administration failed to prevent the formation of these protein modifications in 16-week-old Dusp $1^{-/-}$mice (Figure 4D). Analysis of HO1 and SOD2 protein levels revealed a different pattern of expression to that expected from the mRNA expression data, indicating the likely post-transcriptional regulation of these proteins. Of note, P22PHOX protein levels mirrored the mRNA expression data (Figure 4E), supporting the idea that NAC effectively suppresses NOX-derived $\mathrm{O}_{2}{ }^{--}$generation. Finally, to evaluate pro-apoptotic and survival signaling we studied SAPK and AKT phosphorylated levels, respectively. We observed clear activation of cochlear JNK in NAC-treated Dusp1 $1^{-/-}$mice compared with the other two groups, whereas AKT was slightly activated in untreated Dusp $1^{-/-}$mice as compared with Dusp $1^{+/+}$mice (Figure 4E).

\subsection{N-Acetylcysteine Administration Protects Mitochondria and Restrains DNA Damage in Spiral Ganglion Neurons}

Increased ROS generation and inefficient oxidative phosphorylation are closely linked to impaired mitochondrial function and quality control. Immunolabeling of SGN for mitochondrial complex IV (cytochrome C oxidase)—used as a marker of mitochondrial integrity—revealed evident differences between groups, with significantly stronger neuron immunostaining in NAC-treated Dusp $1^{-/-}$mice, both in the basal and middle cochlear turns, than in untreated Dusp1 $1^{-/-}$mice (Figure 5A,B); no differences were observed between $D u s p 1^{+/+}$and untreated Dusp $1^{-/-}$mice.

To further study energy metabolism and the generation of new mitochondrial mass, we measured the expression levels of the PGC-1 family members (Pgc1 $\alpha, P g c 1 \beta)$, in addition to downstream genes and canonical mitochondrial genes (Figure 5C). Expression data indicated that the mitochondrial biogenesis program was stimulated in 16-week-old $D u s p 1^{+/+}$mice, as evidenced by the higher expression levels of regulatory $(P g c 1 \alpha, P g c 1 \beta$, Ppara, Tfb2m and Alas1) and electron transport chain component (Sdha, Uqcrc2, Mtco1 and Mtap6) genes and citrate synthase (Cs) when compared with younger mice. This induction of gene expression was not evident in NAC-treated or untreated Dusp $1^{-/-}$mice, with the expression of most genes significantly reduced at this age in both groups (Figure 5C). This accords with the downregulation of the PGC1A pathway in Dusp $1^{-/-}$mice revealed by RNAseq (Figure 1).

Redox dysregulation may lead to DNA damage, which was highlighted in the GSEA. To evaluate this, cochlear sections were stained for the DNA damage marker phosphorylated histone 2A.X (p-H2A.X) (Figure 5D), and foci number were quantified in SGN nuclei (Figure 5E). The percentage of SGNs with $>5$ p-H2A.X foci per nucleus was higher in the middle turn cochlea from untreated $D u s p 1^{-/-}$mice than from the other two groups. Of note, p-H2A.X levels in whole cochlear protein extracts were significantly lower in NAC-treated Dusp $1^{-/-}$mice than in untreated Dusp $1^{-/-}$mice (Figure 5F). 
A

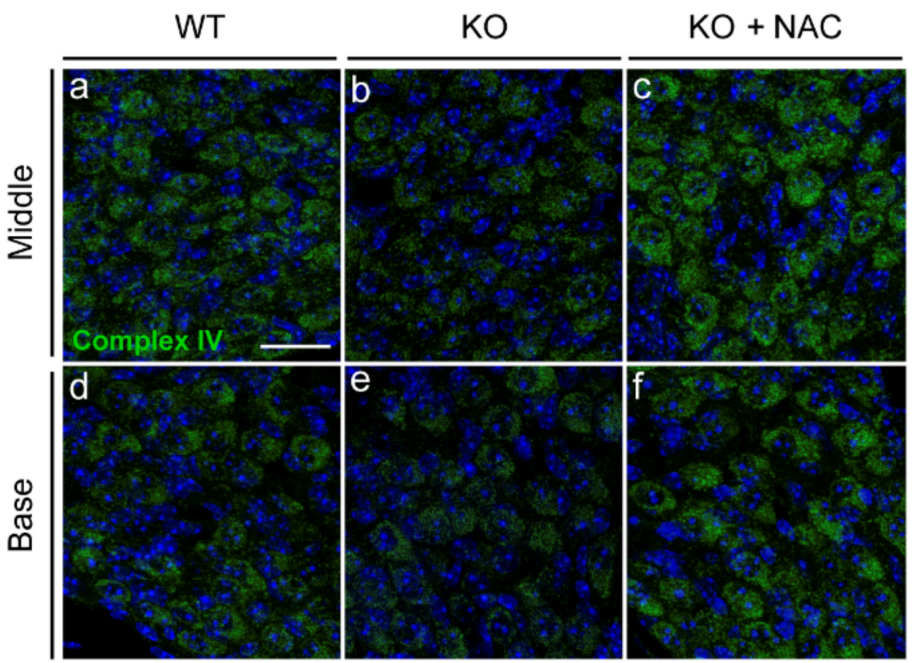

B

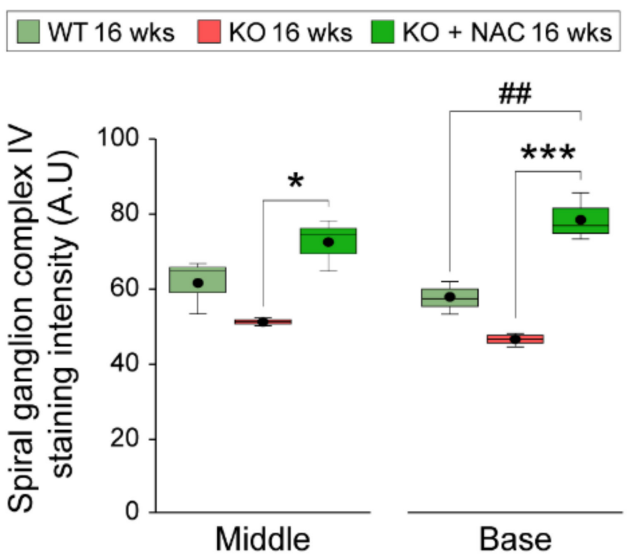

C

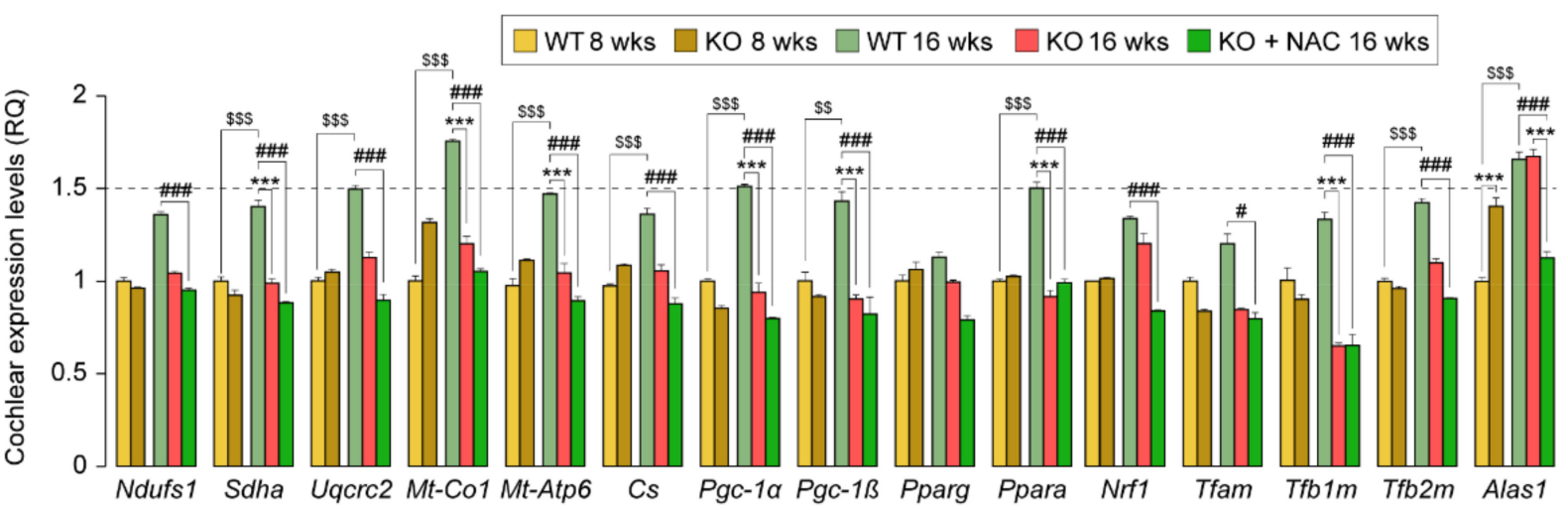

$\mathrm{D}$

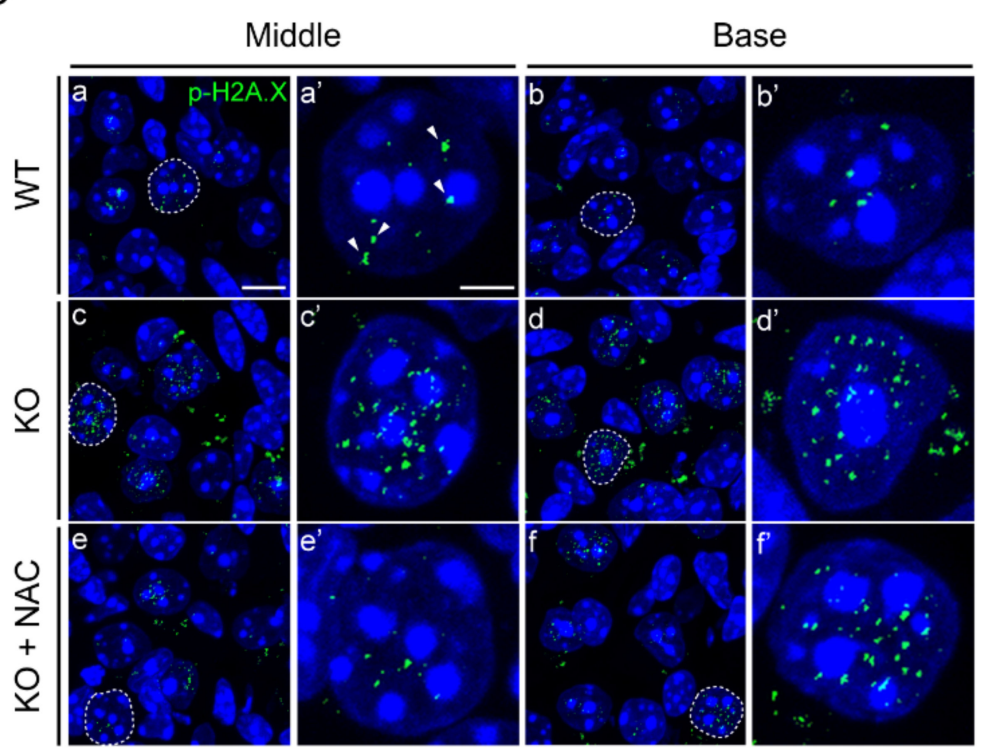

E

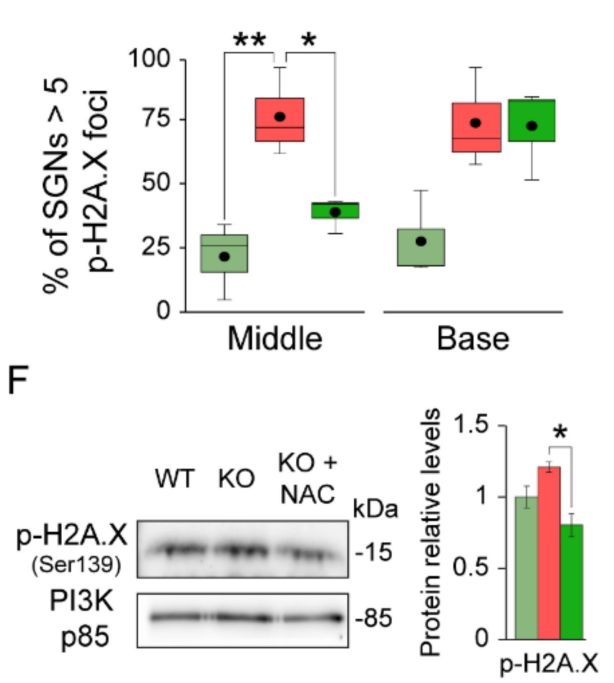

Figure 5. N-acetylcysteine administration preserves mitochondria and reduces DNA damage foci in Dusp1 $1^{-/-}$spiral ganglion neurons. (A) Representative basal and middle turn cochlear spiral ganglion (SG) cross-cryosections of 16-week-old wild-type (WT), Dusp1 knock-out (KO) and NAC-treated Dusp1 KO mice immunolabeled for mitochondrial complex IV (green). Scale bar: $20 \mu \mathrm{m}$ in a. (B) Positive stained area intensity of the SG was quantified in 3 mice per condition in at least 
3 serial sections per turn. Data are presented as a box plot, mean value is plotted as a filled circle and whiskers represent min and max values. (C) RT-qPCR gene expression levels of mitochondrial proteins and biogenesis program genes from whole cochlea pooled samples from 3 mice per condition. Expression levels were calculated as $2^{-\Delta \Delta \mathrm{Ct}}$ (RQ) from triplicate measurements using Hprt1 as a reference gene and normalized to levels in 8-week-old WT mice. Data are presented as mean \pm SEM. (D) Representative basal and middle turn cochlear SG cross-cryosections of 16-week-old WT, Dusp1 KO and NAC-treated Dusp1 KO mice immunolabeled for p-H2A.X in green (a-f). Dashed lines: neuron in close ups (a'-f'). Arrowheads indicate positive foci. Scale bar: $10 \mu \mathrm{m}$ in a and $2.5 \mu \mathrm{m}$ in a'. (E) Percentage of SG neuron (SGN) nuclei with more than 5 p-H2A.X foci. p-H2A.X foci were counted in 5-10 neuronal nuclei per section in 4 serial sections per turn from at least 3 mice per condition. Data are presented as a box plot, mean value is plotted as a filled circle and whiskers represent min and max values. (F) Western blotting of pooled cochlear protein extracts from 3 mice per condition. p-H2A.X data are relative to those for PI3K and normalized to data from 16-week-old WT mice. Data are presented as mean \pm SEM. Statistical significance between genotypes and time points was analyzed by one-way ANOVA: * vs. KO, \# vs. WT, \$ 8-week-old mice

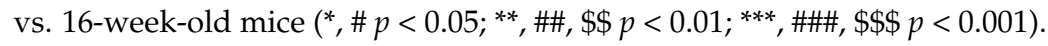

\subsection{N-Acetylcysteine Administration Normalizes the Inflammatory Response and Macrophage Recruitment in Dusp1 ${ }^{-1-}$ Cochlea}

Chronic inflammation is a common feature of aberrant ROS production, which stimulates the inflammatory response in a positive feedback loop to ultimately disrupt cell and tissue homeostasis. In this context, DUSP1 is a key regulator of cell fate in response to oxidative and inflammatory stimuli. To further understand how loss of Dusp 1 impacts the regulation of these two pathways, we studied the production of inflammatory mediators in the 3 groups of mice (Figure 6A). Gene expression analysis of cochleae revealed a marked induction of proinflammatory cytokine gene expression of $\operatorname{Tnf} \alpha, I l 1 \beta, I l 6$ and Il18 in Dusp1 $1^{-/-}$mice from 8 to 16 weeks. Even at the earlier age tested, cytokine expression levels were higher in $D u s p 1^{-/-}$mice than in $D u s p 1^{+/+}$mice with the exception of Il18. Il1 $\beta$ expression also increased from 8 to 16 weeks in $D u s p 1^{+/+}$mice, albeit to a much lesser extent. Of note, cytokine expression levels were similar between NAC-treated $D u s p 1^{-/-}$mice and Dusp $1^{+/+}$mice, indicating that NAC blunts the inflammatory response. A similar NAC response was observed for the expression levels of Tgf $\beta 1, M p o$, Foxp3 and Kim1. Overall, these results suggest that the induction of inflammatory gene expression observed in Dusp $1^{-/-}$cochleae was secondary to the oxidative imbalance, as it could be repressed, albeit incompletely, with NAC. To further confirm this, we tested for the presence of macrophages in the spiral ligament of cochlear sections by IBA1 immunolabeling (Figure 6B). Results showed stronger IBA1 staining in Dusp $1^{-/-}$basal and middle cochlear turns than in equivalent sections from $D u s p 1^{+/+}$mice. NAC administration effectively limited macrophage infiltration in the middle turn but not in the basal turn (Figure 6C), which correlates well with our data on cochlear damage and hearing thresholds. 
A

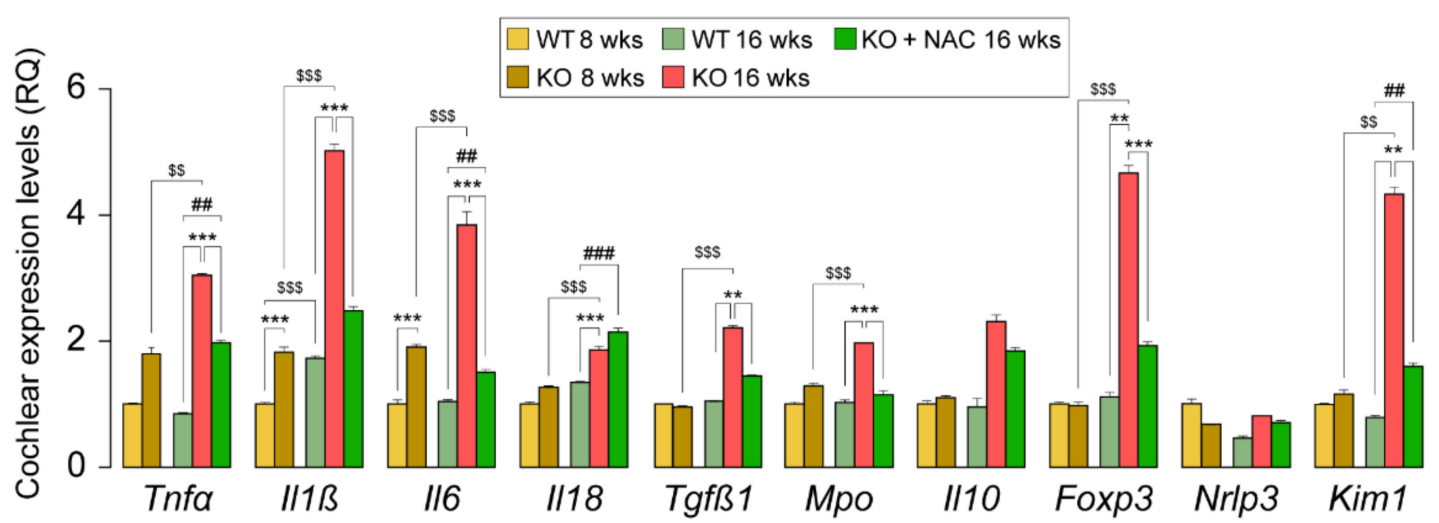

B

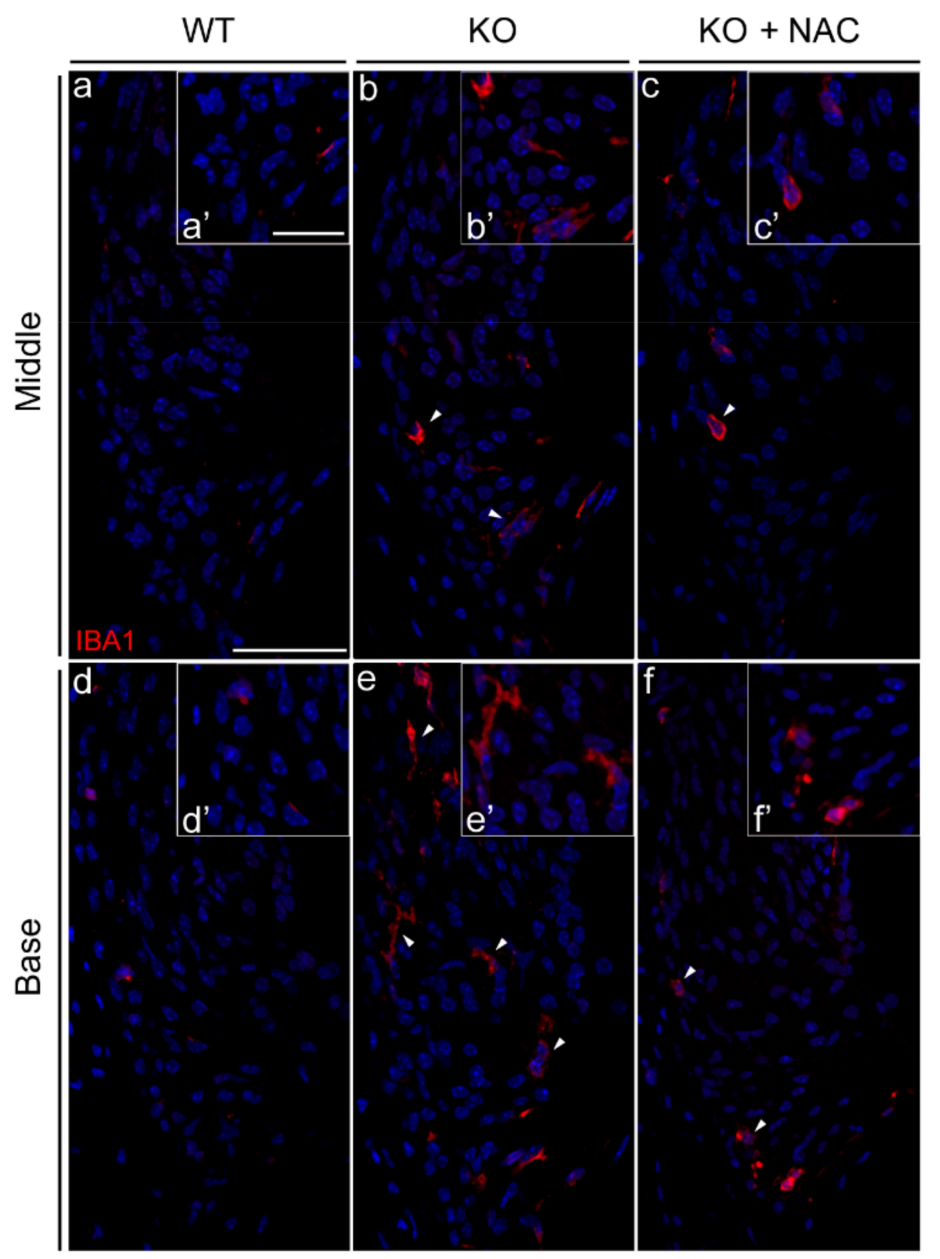

C
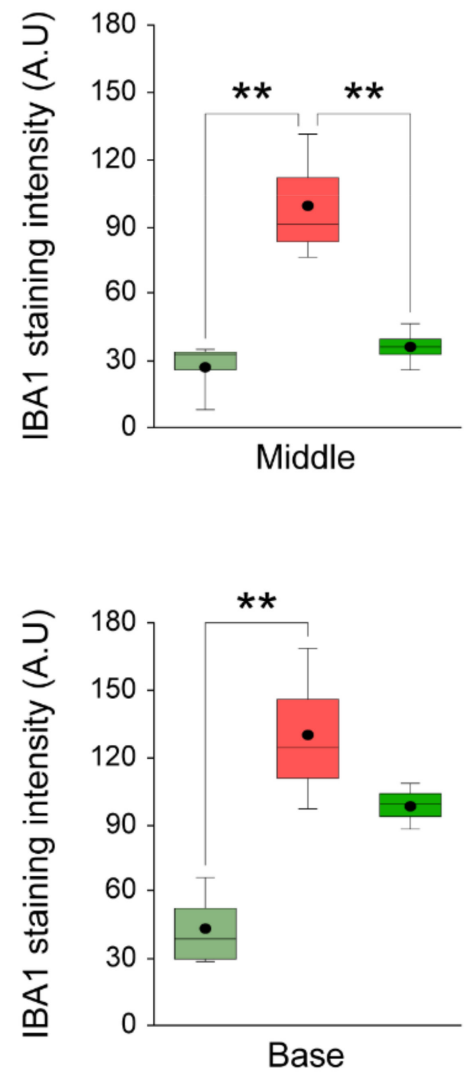

Figure 6. Cochlear exacerbated inflammatory response and macrophage recruitment in $D u s p 1^{-/-}$mice is limited by $\mathrm{N}$-acetylcysteine administration. (A) RT-qPCR gene expression levels of inflammatory mediators from whole cochlea pooled samples from 3 mice per condition. Expression levels were calculated as $2^{-\Delta \Delta C t}(R Q)$ from triplicate measurements using Hprt1 as a reference gene and normalized to levels in 8-week-old wild-type (WT) mice. Data are presented as mean \pm SEM. (B) Representative basal and middle turn cochlear spiral ligament (Spl) cross-cryosections of 16-week-old WT, Dusp1 knock-out (KO) and NAC-treated Dusp1 KO mice immunolabeled for IBA1 in red (a-f). Arrowheads highlight positive macrophages staining. Spl close ups $\left(a^{\prime}-f^{\prime}\right)$. Scale bar: $40 \mu \mathrm{m}$ in a and $25 \mathrm{a}^{\prime}$. (C) IBA1 quantification in the spiral ligament. Staining intensity was quantified in 3 mice per condition in at least 3 serial sections per turn. Data are presented as a box plot, mean value is plotted as a filled circle and whiskers represent min and max values. Statistical significance between genotypes and time points was analyzed by one-way ANOVA: * vs. KO, \# vs. WT, \$ 8-week-old mice vs. 16-week-old mice $(* *, \# \#, \$ p<0.01 ; * * *$, \#\#, \$\$ $p<0.001)$. 


\section{Discussion}

DUSP1 appears to be a converging node for stress-related inflammation and oxidative stress pathways in the cochlea. DUSP1 contributes to redox homeostasis, inflammatory response and, consequently, to hearing preservation. Our findings indicate that administration of the antioxidant NAC to Dusp1-deficient mice mitigates, at least in part, the adverse effects caused by DUSP1 loss-of-function and delays the onset of hearing loss. It is likely, however, that DUSP1 has broader functions, and thus NAC administration fails to fully restore hearing in Dusp1-deficient mice.

Cochlear stress triggers the activation of pro-apoptotic and survival signaling pathways through MAPK cascades [9,39], and the magnitude of cross-talk between these pathways likely tips the balance in one direction or the other [40]. A uniqueness of DUSP1 is its ability to control multiple pathways and to orchestrate cellular responses to multiple stimuli [15]. Our high-throughput cochlear transcriptome profiling provides valuable data on the contribution of DUSP1 to the molecular mechanisms underlying hearing loss. As expected, pathways related to immune system were among the most affected by Dusp 1 deletion in mice, both at 20 and 32 weeks of age. Pro-inflammatory cytokines activate SAPK signaling pathways during the acute phase of the inflammatory response, which can ultimately trigger apoptosis. In this line, tumor necrosis factor- $\alpha(\mathrm{TNF} \alpha)$ is one of the most investigated factors involved in HC death pathways in vivo and in vitro-either by the intrinsic mitochondrial cascade or through the binding of ligands to cell surface death receptors (extrinsic pathway) [41]. Concomitant with immune cell recruitment, the expression of TNF $\alpha$ increases rapidly after cochlear damage, activating nuclear factor kappa-light-chain-enhancer of activated $\mathrm{B}$ cells $(\mathrm{NF}-\mathrm{k} \beta$ ) and stimulating the production of other cytokines, chemokines and adhesion molecules [42]. TNF $\alpha$ and NF- $k \beta$ signaling, cytokine, chemokine, cell adhesion, phagocytosis and pathways related to intrinsic and extrinsic apoptotic cascades were prominent in the enriched pathways in cochleae from 20-week-old Dusp $1^{-/-}$mice. We speculate that $D u s p 1$ deletion renders the cochlea unable to resolve the pro-inflammatory response, contributing to HC and SGN apoptosis $[9,17]$.

The importance of DUSP1 for neuronal homeostasis was also evident in our study, as illustrated by the downregulation of neuronal system pathways in Dusp1 $1^{-/-}$cochleae. Neuroprotective roles have previously been ascribed to DUSP1, and reduced levels have been related to neurological disorders [43]. Our data show that several pathways related to neurodegenerative diseases (including Huntington's, Alzheimer's and Parkinson's disease) were enriched in the absence of DUSP1. Pathways related to DNA processes were also conspicuous in Dusp1 $1^{-/-}$cochlea. Among them, DNA damage pathways including DNA repair, ATM and ATR-BRCA pathways were upregulated. Because of its capacity to inhibit apoptotic signals, DUSP1 has been widely studied in vivo and in vitro as a proto-oncogene and is associated with tumor progression in several types of cancer [44], which agrees with the changes in cell cycle and cancer-related pathways in our dataset.

Mitochondrial function and maintenance of energetic integrity are essential for cellular homeostasis. Based on our data, mitochondrial quality control and biogenesis regulation in the cochlea are likely to be impaired in the absence of DUSP1 [45], as revealed by the downregulation of PGC1 $\alpha$ and the enhanced oxidative phosphorylation pathways in 20week-old Dusp $1^{-/-}$cochlea. Mitochondria are extremely dynamic and environmentally sensitive organelles that can adapt rapidly to adverse conditions to preserve energetic supply [46]. Even mild mitochondrial stress has been proposed to protect the cell through increased ROS signaling as an adaptative response to reduce vulnerability to additional metabolic anomalies [47]. Indeed, mitochondria produce up to the $90 \%$ of all intracellular ROS, which are associated with cellular respiration, making them one of the main targets of oxidative damage. Loss of DUSP1 function might render cochlear cells incapable of adapting to a pro-oxidant environment, leading to overwhelmed mitochondria and damage [48]. This is consistent with the sustained gene enrichment for GSH metabolism observed in 20- and 32-week-old Dusp $1^{-/-}$mice, likely induced to restore redox balance. In this sense, metabolism and inflammation are both closely linked to ROS signaling [49], 
which might account for the exacerbated inflammatory response observed in Dusp1 $1^{-/-}$ mice. These findings suggest that increased ROS production and mitochondrial dysfunction trigger the inflammation and apoptotic pathways activated in Dusp $1^{-/-}$cochlea $[9,39,50]$. This is supported by our finding that the administration of a GSH prodrug restores redox balance and lessens the impact of DUSP1 loss-of-function for cochlear homeostasis.

In anticipation of the oxidative imbalance in $D u s p 1^{-/-}$mice, we administered NAC to pups after weaning. Measurement of GSH levels indicated that the usage of GSH is finely tuned in NAC-treated cochleae, with higher total GSH levels and lower GSR turnover rate than in untreated cochleae, suggesting a reduced need for replacing GSSG to increase the GSH pool. Accordingly, Ggt1 mRNA levels were reduced, which might indicate that NAC provides a more efficient source of cysteine than the ATP-dependent process associated with GSH extracellular export and recycling. Indeed, NAC administration has been reported before to prevent progressive hearing loss in Ggt1 deficient mice, presumably by providing cysteine [51].

In line with the above, we found that the expression of antioxidant defense enzymes was not induced in NAC-treated cochlea samples, and no differences were observed in protein carbonylation. It is likely that the correct combination of antioxidants with different mechanisms of action, rather than NAC alone, would enhance the cochlear antioxidant defense impacting simultaneously at different levels [5]. By contrast, WT cochleae showed an age-related and coordinated induction of antioxidant genes, possibly driven by NRF2 [52], which prevented protein oxidative modifications. However, our results point to a limitation in ROS production at this age that correlated with a better preservation of mitochondrial function and energetic supply in NAC-treated cochlea. Conversely, NADPH production by G6PD and PGD was not reduced in NAC-treated cochlea, which is particularly intriguing as NOX and GSR activities depend highly on this cofactor [53]. In this regard, RNAseq data suggest that the distinct regulation of G6PD and PGD in wild type and knockout mice may be a consequence of the metabolic alterations in the pentose phosphate cycle associated with Dusp1 deficiency. Furthermore, Dusp $1^{-/-}$ cochlea showed increased transcript and protein levels of P22PHOX [25], suggesting that NADPH production could be increased to maintain the levels due to increased spend. In contrast, we speculate that neither $\mathrm{O}_{2}{ }^{-}$generation (by NOX) nor GSH regeneration is required at higher rates in NAC-treated cochlea. Therefore, NADPH is likely used by other NADPH-dependent enzymes that participate in reductive anabolic reactions.

The mitochondrial biogenesis program was not activated in NAC-treated Dusp1 $1^{-/-}$ mice in contrast to what we observed in WT cochleae. Nevertheless, mitochondria integrity was preserved in NAC-treated SGNs. On this basis, we propose that early NAC administration generates a balanced redox environment in the mitochondria that prevents the oxidative damage in the organelle [54] that would, otherwise, have a detrimental outcome, as observed in untreated Dusp $1^{-/-}$mice.

Active SAPKs promote cytokine production and leukocyte migration to injury sites. Therefore, tight control of the activation of these kinases is key to preventing an inflammatory response and the propagation of damage [17]. In this context, DUSP1 is induced in response to inflammatory stimuli to mediate shutdown of the acute inflammation phase [55]. We found that inflammation was present at early stages in $D u s p 1^{-/-}$cochlea, later becoming chronic with production of inflammatory mediators that contributed to severity and propagation of damage accompanied by increased macrophage recruitment [56,57]. In this regard, expression of the master regulator of the development and function of regulatory T-cells, Foxp3, was induced in Dusp1 $1^{-1-}$ cochlea at 16 weeks of age. When stimulated, regulatory T-cells aid in resolving established inflammation by suppressing immune responses of other cells and inducing them to express immunosuppressive cytokines such as IL-10 [58]. Despite the higher Il10 expression in 16-week-old Dusp $1^{-/-}$mice, the increase was not significant and might indicate deficient activation of the inflammation resolution phase. Specifically, IL-10 is known to induce the robust expression of Dusp1 that sustains 
mRNA levels in a feedback loop and correlates with inhibition of p38 signaling and with IL-6 and IL-12 production in vitro [59].

Inflammation was reduced in NAC-treated mice, although it was not completely neutralized, as illustrated by increased Tnf $\alpha$, Il6 and Il18 expression levels. It is possible that NAC contributes to preventing early ROS-driven pro-inflammatory cytokine production and the later chronic inflammatory state $[60,61]$, as observed in NAC-treated 16-week-old Dusp $1^{-/-}$. Nevertheless, the cellular antioxidant system is complex, and while glutathione is the most powerful cellular antioxidant, it represents just one of the several lines of defense against ROS. In this context, the impact of NAC administration is limited and might explain the higher JNK activation levels [62] observed in NAC-treated 16-weekold Dusp $1^{-/-}$mice, which may have occurred earlier in chronically inflamed untreated Dusp $1^{-/-}$cochlea. We would suggest that HC loss may spread quickly in NAC-treated mice, emulating the situation in untreated mice and supporting the idea that hearing loss onset is lessened but not prevented by NAC. Indeed, although much lower than in untreated mice, the gene expression of the damage biomarker kidney injury molecule 1 (KIM-1) [9] was higher in NAC-treated mice than in control mice, which also points in this direction. Cochlear levels of KIM-1 have been reported to increase following NOX activation and ROS generation, and ROS scavengers and NOX inhibitors attenuated this increase [63]. Notably, Kim1 induction upon ROS generation by NOX was also observed in the Dusp $1^{-1-}$ cochlea as shown by increased P22PHOX mRNA and protein levels, which were inhibited by treatment with NAC.

Independent of ROS production, aberrant regulation of inflammation due to DUSP1 loss-of-function contributes to damage extension and hearing loss. Indeed, corticoid treatment was previously found to reduce TNF $\alpha$ levels in the spiral ligament and activate survival pathways in HCs, inhibiting JNK signaling [64]. In this context, modulation of both mechanisms simultaneously could be a more robust strategy to ameliorate the metabolic disturbances in Dusp $1^{-/-}$cochlea. Indeed, there is evidence showing that drug combination approaches attenuate human hearing loss [65]. In fact, protection from oxidative stress and limited HC death were reported in patients with sudden SNHL by combined NAC-dexamethasone treatment, but both compounds were ineffective when administered alone [66].

Overall, our study provides evidence to consider pharmacological modulation of DUSP1 activity as a potential treatment for hearing loss, as an alternative to current SAPK inhibitors $[13,14,67,68]$. Nevertheless, given its unique physiological activity, DUSP1 induction and activity regulation is finely tuned, and so treatments would be highly dependent on the correct timing and kinetics of both DUSP1 and the proteins involved in the signaling pathways. It is clear that several issues need to be addressed and numerous challenges overcome to achieve the successful modulation of DUSP1 activity [69].

\section{Conclusions}

We provide new insight into the role of DUSP1 in the cochlea using a combination of transcriptomics and antioxidant treatment of Dusp1-deficient mice. We present here, for the first time, transcriptome sequencing data from cochleae suffering unrestrained cellular stress due to the absence of its natural regulator DUSP1. Our data improve knowledge on the basic molecular mechanisms that orchestrate the regulation of oxidative stress, inflammation and mitochondrial quality control in the ageing cochlea. The molecular mechanisms underlying SNHL have multiple common aspects; thus, this work provides information beyond ARHL. Finally, our findings point to DUSP1 as a potentially druggable target to impair HC and SGN death in response to stress.

Supplementary Materials: The following are available online at https://www.mdpi.com/article/10 .3390/antiox10091351/s1, Figure S1: Comparative hearing loss progression and sound conduction efficiency of Dusp $1^{+/+}, D u s p 1^{-/-}$and N-acetylcysteine-treated Dusp $1^{-/-}$mice; Table S1: Gene sets enriched in Dusp1-/- cochlea; Table S2: TaqMan probes and primers for RT-qPCR experiments. 
Author Contributions: Conceptualization, J.M.B.-M., A.M.C., M.S., I.V.-N.; Methodology, J.M.B.-M., A.M.C., M.S., I.V.-N.; Software, J.M.B.-M., A.M.C., D.M.-E.; Validation, J.M.B.-M., A.M.C., Á.G.-M., I.V.-N.; Formal Analysis J.M.B.-M., Á.G.-M., D.M.-E.; Investigation, J.M.B.-M., A.M.C., Á.G.-M., L.R.-d.1.R.; Resources, J.M.B.-M., L.R.-d.1.R., D.M.-E.; Data curation, J.M.B.-M., A.M.C., Á.G.-M., L.R.-d.1.R.; Writing-Original Draft Preparation, J.M.B.-M., I.V.-N.; Writing-Review and Editing, J.M.B.-M., A.M.C., Á.G.-M., L.R.-d.1.R., M.S., I.V.-N.; Visualization, J.M.B.-M., I.V.-N.; Supervision, J.M.B.-M., I.V.-N.; Project administration, I.V.-N.; Funding Acquisition, M.S., I.V.-N. All authors have read and agreed to the published version of the manuscript.

Funding: This work was funded by FEDER/B2017/BMD-3688-MULTITARGET\&VIEWCM and 0551-PSL-6-E NITROPROHEAR grants to I.V.-N., J.M.B.-M., Á.G.-M. and L.R.-d.1.R. held SAF2017HEARCODE, FPU (FPU16/03308; MECD) and CIBERER (Institute of Health Carlos III) contracts, respectively, co-financed with FEDER funds. Work by D.M.-E. and M.S. was funded by grant SAF201782613-R from the Spanish Ministry of Science co-funded by the European Regional Development Fund (ERDF). Work in the laboratory of M.S. was also funded by the IRB and "laCaixa" Foundation, and by grants from the European Research Council (ERC-2014-AdG/669622), and Secretaria d'Universitats i Recerca del Departament d'Empresa i Coneixement of Catalonia (Grup de Recerca consolidat 2017 SGR 282. D.M.-E. laboratory is supported by the Cancer Research UK (CRUK) Cambridge Centre Early Detection Programme (RG86786), by a CRUK Programme Foundation Award (C62187/A29760), by a CRUK Early Detection OHSU Project Award (C62187/A26989), and by a Medical Research Council (MRC) New Investigator Research Grant (NIRG) (MR/R000530/1).

Institutional Review Board Statement: The study was conducted according to the guidelines and approved by the Institutional Review Board (or Ethics Committee) of the Spanish National Research Council (CSIC) and Madrid Community Bioethics Committee, with authorization code PROEX 010/18 (16 February 2018).

Informed Consent Statement: Not applicable.

Data Availability Statement: RNAseq data discussed in this publication have been deposited in NCBI's Gene Expression Omnibus [70] and are accessible through GEO Series accession number GSE176114 (https:/ / www.ncbi.nlm.nih.gov/geo/ query / acc.cgi?acc=GSE176114). Additional data that support the findings of this study are contained within the article.

Acknowledgments: We are grateful to our colleagues of the Neurobiology of Hearing Group and Elisa Zubeldia-Varela (San Pablo-CEU University, Madrid) for their technical support, critical comments and sharing of unpublished information. We also thank the CNIO Bioinformatics Unit, the Histology Unit (CNB, CSIC) and the Non-Invasive Neuro Functional Evaluation and Genomics facilities (IIBM, CSIC-UAM). We thank Kenneth McCreath for his help editing the manuscript.

Conflicts of Interest: M.S. is founder, shareholder and advisor of Senolytic Therapeutics, Inc., Iduna Therapeutics, Inc. and RejuverSen, AG.

\section{References}

1. Gates, G.A.; Mills, J.H. Presbycusis. Lancet 2005, 366, 1111-1120. [CrossRef]

2. WHO. Deafness and Hearing Loss. 2021. Available online: https://www.who.int/news-room/fact-sheets/detail/deafness-andhearing-loss (accessed on 1 March 2021).

3. Prasad, K.N.; Bondy, S.C. Increased oxidative stress, inflammation, and glutamate: Potential preventive and therapeutic targets for hearing disorders. Mech. Ageing Dev. 2020, 185, 111191. [CrossRef] [PubMed]

4. Pak, J.H.; Kim, Y.; Yi, J.; Chung, J.W. Antioxidant Therapy against Oxidative Damage of the Inner Ear: Protection and Preconditioning. Antioxidants 2020, 9, 1076. [CrossRef] [PubMed]

5. García-Alcántara, F.; Murillo-Cuesta, S.; Pulido, S.; Bermúdez-Muñoz, J.M.; Martínez-Vega, R.; Milo, M.; Varela-Nieto, I.; Rivera, T. The expression of oxidative stress response genes is modulated by a combination of resveratrol and $\mathrm{N}$-acetylcysteine to ameliorate ototoxicity in the rat cochlea. Hear. Res. 2018, 358, 10-21. [CrossRef] [PubMed]

6. Aldini, G.; Altomare, A.A.; Baron, G.; Vistoli, G.; Carini, M.; Borsani, L.; Sergio, F. N-Acetylcysteine as an antioxidant and disulphide breaking agent: The reasons why. Free. Radic. Res. 2018, 52, 751-762. [CrossRef] [PubMed]

7. Wang, J.; Puel, J.-L. Toward Cochlear Therapies. Physiol. Rev. 2018, 98, 2477-2522. [CrossRef]

8. López-Otín, C.; Blasco, M.A.; Partridge, L.; Serrano, M.; Kroemer, G. The Hallmarks of Aging. Cell 2013, $153,1194-1217$. [CrossRef] [PubMed] 
9. Celaya, A.M.; Sánchez-Pérez, I.; Bermúdez-Muñoz, J.M.; Rodríguez-de la Rosa, L.; Pintado-Berninches, L.; Perona, R.; MurilloCuesta, S.; Varela-Nieto, I. Deficit of mitogen-activated protein kinase phosphatase 1 (DUSP1) accelerates progressive hearing loss. eLife 2019, 8, 8. [CrossRef]

10. Kyriakis, J.M.; Avruch, J. Mammalian MAPK Signal Transduction Pathways Activated by Stress and Inflammation: A 10-Year Update. Physiol. Rev. 2012, 92, 689-737. [CrossRef]

11. Wang, J.; Van De Water, T.R.; Bonny, C.; De Ribaupierre, F.; Puel, J.-L.; Zine, A. A Peptide Inhibitor of c-Jun N-Terminal Kinase Protects against Both Aminoglycoside and Acoustic Trauma-Induced Auditory Hair Cell Death and Hearing Loss. J. Neurosci. 2003, 23, 8596-8607. [CrossRef]

12. Jamesdaniel, S.; Hu, B.; Kermany, M.H.; Jiang, H.; Ding, D.; Coling, D.; Salvi, R. Noise induced changes in the expression of p38/MAPK signaling proteins in the sensory epithelium of the inner ear. J. Proteom. 2011, 75, 410-424. [CrossRef]

13. Suckfuell, M.; Lisowska, G.; Domka, W.; Kabacinska, A.; Morawski, K.; Bodlaj, R.; Klimak, P.; Kostrica, R.; Meyer, T. Efficacy and Safety of AM-111 in the Treatment of Acute Sensorineural Hearing Loss: A Double-Blind, Randomized, Placebo-Controlled Phase II Study. Otol. Neurotol. 2014, 35, 1317-1326. [CrossRef] [PubMed]

14. Wang, J.; Ruel, J.; Ladrech, S.; Bonny, C.; Van De Water, T.R.; Puel, J.-L. Inhibition of the c-Jun N-Terminal Kinase-Mediated Mitochondrial Cell Death Pathway Restores Auditory Function in Sound-Exposed Animals. Mol. Pharmacol. 2006, 71, 654-666. [CrossRef] [PubMed]

15. Wancket, L.M.; Frazier, W.J.; Liu, Y. Mitogen-activated protein kinase phosphatase (MKP)-1 in immunology, physiology, and disease. Life Sci. 2012, 90, 237-248. [CrossRef] [PubMed]

16. Lawan, A.; Shi, H.; Gatzke, F.; Bennett, A.M. Diversity and specificity of the mitogen-activated protein kinase phosphatase-1 functions. Cell. Mol. Life Sci. 2012, 70, 223-237. [CrossRef]

17. Chi, H.; Barry, S.P.; Roth, R.J.; Wu, J.J.; Jones, E.A.; Bennett, A.M.; Flavell, R.A. Dynamic regulation of pro- and anti-inflammatory cytokines by MAPK phosphatase 1 (MKP-1) in innate immune responses. Proc. Natl. Acad. Sci. USA 2006, 103, 2274-2279. [CrossRef]

18. Kassel, O.; Sancono, A.; Krätzschmar, J.; Kreft, B.; Stassen, M.; Cato, A.C. Glucocorticoids inhibit MAP kinase via increased expression and decreased degradation of MKP-1. EMBO J. 2001, 20, 7108-7116. [CrossRef]

19. Shah, S.; King, E.M.; Chandrasekhar, A.; Newton, R. Roles for the Mitogen-activated Protein Kinase (MAPK) Phosphatase, DUSP1, in Feedback Control of Inflammatory Gene Expression and Repression by Dexamethasone. J. Biol. Chem. 2014, 289, 13667-13679. [CrossRef]

20. Maeda, K.; Yoshida, K.; Ichimiya, I.; Suzuki, M. Dexamethasone inhibits tumor necrosis factor- $\alpha$-induced cytokine secretion from spiral ligament fibrocytes. Hear. Res. 2005, 202, 154-160. [CrossRef]

21. King, E.M.; Holden, N.; Gong, W.; Rider, C.; Newton, R. Inhibition of NF-kB-dependent Transcription by MKP-1: Transcriptional Repression by Glucocorticoids Occurring Via P38 Mapk*. J. Biol. Chem. 2009, 284, 26803-26815. [CrossRef]

22. Dorfman, K.; Carrasco, D.; Gruda, M.; Ryan, C.; A Lira, S.; Bravo, R. Disruption of the erp/mkp-1 gene does not affect mouse development: Normal MAP kinase activity in ERP/MKP-1-deficient fibroblasts. Oncogene 1996, 13, 925-931. [PubMed]

23. Marie, A.; Meunier, J.; Brun, E.; Malmstrom, S.; Baudoux, V.; Flaszka, E.; Naert, G.; Roman, F.; Cosnier-Pucheu, S.; GonzalezGonzalez, S. N-acetylcysteine Treatment Reduces Age-related Hearing Loss and Memory Impairment in the SenescenceAccelerated Prone 8 (SAMP8) Mouse Model. Aging Dis. 2018, 9, 664-673. [CrossRef] [PubMed]

24. Cediel, R.; Riquelme, R.; Contreras, J.; Díaz, A.; Varela-Nieto, I. Sensorineural hearing loss in insulin-like growth factor I-null mice: A new model of human deafness. Eur. J. Neurosci. 2006, 23, 587-590. [CrossRef] [PubMed]

25. Bermúdez-Muñoz, J.M.; Celaya, A.M.; Hijazo-Pechero, S.; Wang, J.; Serrano, M.; Varela-Nieto, I. G6PD overexpression protects from oxidative stress and age-related hearing loss. Aging Cell 2020, 19, e13275. [CrossRef]

26. Trapnell, C.; Roberts, A.; Goff, L.; Pertea, G.; Kim, D.; Kelley, D.R.; Pimentel, H.; Salzberg, S.L.; Rinn, J.L.; Pachter, L. Differential gene and transcript expression analysis of RNA-seq experiments with TopHat and Cufflinks. Nat. Protoc. 2012, 7, 562-578. [CrossRef]

27. Langmead, B.; Trapnell, C.; Pop, M.; Salzberg, S.L. Ultrafast and memory-efficient alignment of short DNA sequences to the human genome. Genome Biol. 2009, 10, R25. [CrossRef]

28. Li, H.; Handsaker, B.; Wysoker, A.; Fennell, T.; Ruan, J.; Homer, N.; Marth, G.; Abecasis, G.; Durbin, R. The Sequence Alignment/Map format and SAMtools. Bioinformatics 2009, 25, 2078-2079. [CrossRef]

29. Subramanian, A.; Tamayo, P.; Mootha, V.K.; Mukherjee, S.; Ebert, B.L.; Gillette, M.A.; Paulovich, A.; Pomeroy, S.L.; Golub, T.R.; Lander, E.S.; et al. Gene set enrichment analysis: A knowledge-based approach for interpreting genome-wide expression profiles. Proc. Natl. Acad. Sci. USA 2005, 102, 15545-15550. [CrossRef]

30. Merico, D.; Isserlin, R.; Stueker, O.; Emili, A.; Bader, G.D. Enrichment Map: A Network-Based Method for Gene-Set Enrichment Visualization and Interpretation. PLoS ONE 2010, 5, e13984. [CrossRef]

31. Kucera, M.; Isserlin, R.; Arkhangorodsky, A.; Bader, G.D. AutoAnnotate: A Cytoscape app for summarizing networks with semantic annotations. F1000Research 2016, 5, 1717. [CrossRef]

32. Cytoscape: A Software Environment for Integrated Models of Biomolecular Interaction Networks-PubMed. Available online: https:/ / pubmed.ncbi.nlm.nih.gov/14597658/ (accessed on 1 March 2021). 
33. Reimand, J.; Isserlin, R.; Voisin, V.; Kucera, M.; Tannus-Lopes, C.; Rostamianfar, A.; Wadi, L.; Meyer, M.; Wong, J.; Xu, C.; et al. Pathway enrichment analysis and visualization of omics data using g:Profiler, GSEA, Cytoscape and EnrichmentMap. Nat. Protoc. 2019, 14, 482-517. [CrossRef] [PubMed]

34. Babicki, S.; Arndt, D.; Marcu, A.; Liang, Y.; Grant, J.R.; Maciejewski, A.; Wishart, D.S. Heatmapper: Web-enabled heat mapping for all. Nucleic Acids Res. 2016, 44, W147-W153. [CrossRef]

35. Sanchez-Calderon, H.; Rodriguez-de la Rosa, L.; Milo, M.; Pichel, J.; Holley, M.; Varela-Nieto, I. RNA Microarray Analysis in Prenatal Mouse Cochlea Reveals Novel IGF-I Target Genes: Implication of MEF2 and FOXM1 Transcription Factors. PLoS ONE 2010, 5, e8699. [CrossRef]

36. Schindelin, J.; Arganda-Carreras, I.; Frise, E.; Kaynig, V.; Longair, M.; Pietzsch, T.; Preibisch, S.; Rueden, C.; Saalfeld, S.; Schmid, B.; et al. Fiji: An open-source platform for biological-image analysis. Nat. Methods 2012, 9, 676-682. [CrossRef]

37. White, K.; Kim, M.-J.; Ding, D.; Han, C.; Park, H.-J.; Meneses, Z.; Tanokura, M.; Linser, P.; Salvi, R.; Someya, S. G6pd Deficiency Does Not Affect the Cytosolic Glutathione or Thioredoxin Antioxidant Defense in Mouse Cochlea. J. Neurosci. 2017, 37, 5770-5781. [CrossRef] [PubMed]

38. Rahman, I.; Kode, A.; Biswas, S.K. Assay for quantitative determination of glutathione and glutathione disulfide levels using enzymatic recycling method. Nat. Protoc. 2006, 1, 3159-3165. [CrossRef]

39. Wong, A.C.; Ryan, A.F. Mechanisms of sensorineural cell damage, death and survival in the cochlea. Front. Aging Neurosci. 2015, 7, 58. [CrossRef] [PubMed]

40. Johnson, G.L.; Lapadat, R. Mitogen-Activated Protein Kinase Pathways Mediated by ERK, JNK, and p38 Protein Kinases. Science 2002, 298, 1911-1912. [CrossRef]

41. Deng, Y.; Ren, X.; Yang, L.; Lin, Y.; Wu, X. A JNK-Dependent Pathway Is Required for TNF $\alpha$-Induced Apoptosis. Cell 2003, 115, 61-70. [CrossRef]

42. Dinh, C.T.; Goncalves, S.; Bas, E.; Van De Water, T.R.; Zine, A. Molecular regulation of auditory hair cell death and approaches to protect sensory receptor cells and/or stimulate repair following acoustic trauma. Front. Cell. Neurosci. 2015, 9, 96. [CrossRef]

43. Pérez-Sen, R.; Queipo, M.J.; Gil-Redondo, J.C.; Ortega, F.; Gómez-Villafuertes, R.; Miras-Portugal, M.T.; Delicado, E.G. DualSpecificity Phosphatase Regulation in Neurons and Glial Cells. Int. J. Mol. Sci. 2019, 20, 1999. [CrossRef]

44. Bermudez, O.; Pagès, G.; Gimond, C. The dual-specificity MAP kinase phosphatases: Critical roles in development and cancer. Am. J. Physiol. Physiol. 2010, 299, C189-C202. [CrossRef]

45. Bauerfeld, C.; Talwar, H.; Zhang, K.; Liu, Y.; Samavati, L. MKP-1 Modulates Mitochondrial Transcription Factors, Oxidative Phosphorylation, and Glycolysis. ImmunoHorizons 2020, 4, 245-258. [CrossRef]

46. Manoli, I.; Alesci, S.; Blackman, M.R.; Su, Y.A.; Rennert, O.M.; Chrousos, G.P. Mitochondria as key components of the stress response. Trends Endocrinol. Metab. 2007, 18, 190-198. [CrossRef] [PubMed]

47. Yun, J.; Finkel, T. Mitohormesis. Cell Metab. 2014, 19, 757-766. [CrossRef]

48. Di Meo, S.; Reed, T.T.; Venditti, P.; Victor, V. Role of ROS and RNS Sources in Physiological and Pathological Conditions. Oxidative Med. Cell. Longev. 2016, 2016, 1-44. [CrossRef]

49. Mittal, M.; Siddiqui, M.R.; Tran, K.; Reddy, S.P.; Malik, A.B. Reactive Oxygen Species in Inflammation and Tissue Injury. Antioxid. Redox Signal. 2014, 20, 1126-1167. [CrossRef]

50. Blaser, H.; Dostert, C.; Mak, T.W.; Brenner, D. TNF and ROS Crosstalk in Inflammation. Trends Cell Biol. 2016, $26,249-261$. [CrossRef] [PubMed]

51. Ding, D.; Jiang, H.; Chen, G.-D.; Longo-Guess, C.; Muthaiah, V.P.K.; Tian, C.; Sheppard, A.; Salvi, R.; Johnson, K.R. N-acetylcysteine prevents age-related hearing loss and the progressive loss of inner hair cells in $\gamma$-glutamyl transferase 1 deficient mice. Aging 2016, 8, 730-750. [CrossRef]

52. Li, D.; Zhao, H.; Cui, Z.-K.; Tian, G. The Role of Nrf2 in Hearing Loss. Front. Pharmacol. 2021, 12, 620921. [CrossRef] [PubMed]

53. Xiao, W.; Wang, R.-S.; Handy, D.E.; Loscalzo, J. NAD(H) and NADP(H) Redox Couples and Cellular Energy Metabolism. Antioxid. Redox Signal. 2018, 28, 251-272. [CrossRef]

54. Calabrese, G.; Morgan, B.; Riemer, J. Mitochondrial Glutathione: Regulation and Functions. Antioxid. Redox Signal. 2017, 27, 1162-1177. [CrossRef]

55. Perdiguero, E.; Sousa-Victor, P.; Ruiz-Bonilla, V.; Jardí, M.; Caelles, C.; Serrano, A.L.; Muñoz-Cánoves, P. p38/MKP-1-regulated AKT coordinates macrophage transitions and resolution of inflammation during tissue repair. J. Cell Biol. 2011, 195, 307-322. [CrossRef]

56. Noble, K.V.; Liu, T.; Matthews, L.J.; Schulte, B.A.; Lang, H. Age-Related Changes in Immune Cells of the Human Cochlea. Front. Neurol. 2019, 10, 895. [CrossRef] [PubMed]

57. Landegger, L.D.; Vasilijic, S.; Fujita, T.; Soares, V.Y.; Seist, R.; Xu, L.; Stankovic, K.M. Cytokine Levels in Inner Ear Fluid of Young and Aged Mice as Molecular Biomarkers of Noise-Induced Hearing Loss. Front. Neurol. 2019, 10, 977. [CrossRef]

58. Campbell, D.J.; Koch, M.A. Phenotypical and functional specialization of FOXP3+ regulatory T cells. Nat. Rev. Immunol. 2011, 11, 119-130. [CrossRef]

59. Hammer, M.; Mages, J.; Dietrich, H.; Schmitz, F.; Striebel, F.; Murray, P.J.; Wagner, H.; Lang, R. Control of dual-specificity phosphatase-1 expression in activated macrophages by IL-10. Eur. J. Immunol. 2005, 35, 2991-3001. [CrossRef] [PubMed]

60. Tirouvanziam, R.; Conrad, C.K.; Bottiglieri, T.; Herzenberg, L.A.; Moss, R.B. High-dose oral N-acetylcysteine, a glutathione prodrug, modulates inflammation in cystic fibrosis. Proc. Natl. Acad. Sci. USA 2006, 103, 4628-4633. [CrossRef] 
61. Pathak, S.; Stern, C.; Vambutas, A. N-Acetylcysteine attenuates tumor necrosis factor alpha levels in autoimmune inner ear disease patients. Immunol. Res. 2015, 63, 236-245. [CrossRef] [PubMed]

62. Kamata, H.; Honda, S.-I.; Maeda, S.; Chang, L.; Hirata, H.; Karin, M. Reactive Oxygen Species Promote TNF $\alpha$-Induced Death and Sustained JNK Activation by Inhibiting MAP Kinase Phosphatases. Cell 2005, 120, 649-661. [CrossRef]

63. Mukherjea, D.; Whitworth, C.; Nandish, S.; Dunaway, G.; Rybak, L.; Ramkumar, V. Expression of the kidney injury molecule 1 in the rat cochlea and induction by cisplatin. Neuroscience 2006, 139, 733-740. [CrossRef]

64. Dinh, C.; Haake, S.; Chen, S.; Hoang, K.; Nong, E.; Eshraghi, A.; Balkany, T.; Van De Water, T. Dexamethasone protects organ of corti explants against tumor necrosis factor-alpha-induced loss of auditory hair cells and alters the expression levels of apoptosis-related genes. Neuroscience 2008, 157, 405-413. [CrossRef] [PubMed]

65. Schreiber, B.E.; Agrup, C.; Haskard, D.O.; Luxon, L.M. Sudden sensorineural hearing loss. Lancet 2010, 375, 1203-1211. [CrossRef]

66. Bai, X.; Chen, S.; Xu, K.; Jin, Y.; Niu, X.; Xie, L.; Qiu, Y.; Liu, X.-Z.; Sun, Y. N-Acetylcysteine Combined With Dexamethasone Treatment Improves Sudden Sensorineural Hearing Loss and Attenuates Hair Cell Death Caused by ROS Stress. Front. Cell Dev. Biol. 2021, 9, 659486. [CrossRef]

67. Doddareddy, M.R.; Rawling, T.; Ammit, A. Targeting mitogen-activated protein kinase phosphatase-1 (MKP-1): Structure-based design of MKP-1 inhibitors and upregulators. Curr. Med. Chem. 2012, 19, 163-173. [CrossRef] [PubMed]

68. Eshraghi, A.A.; Hoosien, G.; Ramsay, S.; Dinh, C.T.; Bas, E.; Balkany, T.J.; Van De Water, T.R. Inhibition of the JNK Signal Cascade Conserves Hearing Against Electrode Insertion Trauma-Induced Loss. Cochlea Implant. Int. 2010, 11, 104-109. [CrossRef] [PubMed]

69. Hoppstädter, J.; Ammit, A. Role of Dual-Specificity Phosphatase 1 in Glucocorticoid-Driven Anti-inflammatory Responses. Front. Immunol. 2019, 10, 1446. [CrossRef]

70. Edgar, R.; Domrachev, M.; Lash, A.E. Gene Expression Omnibus: NCBI gene expression and hybridization array data repository. Nucleic Acids Res. 2002, 30, 207-210. [CrossRef] 